Scientia Agricola

http://dx.doi.org/10.1590/1678-992X-2016-0372

\title{
Lime and calcium-magnesium silicate in the ionic speciation of an Oxisol
}

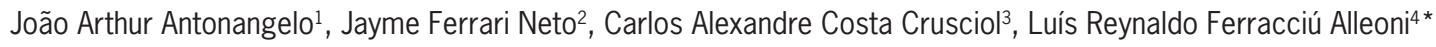

${ }^{1}$ Oklahoma State University - Dept. of Plant and Soil Sciences, 039 Agricultural Hall - 74078 - Stillwater, OK USA.

${ }^{2}$ Sociedade Porvir Científico - La Salle Faculty, Av. Universitária, 1000w - Bairro Bandeirantes - 78455-000 Lucas do Rio Verde, MT - Brazil.

${ }^{3}$ São Paulo State University/FCA - Dept. of Crop Science, R. José Barbosa de Barros, 1780 - 18610-307 - Botucatu, SP - Brazil.

4University of São Paulo/ESALQ - Dept. of Soil Science, Av. Pádua Dias, 11 - 13418-900 - Piracicaba, SP - Brazil.

*Corresponding author <alleoni@usp.br>

Edited by: Leônidas Carrijo Azevedo Melo

Received July 13, 2016

Accepted October 07, 2016
ABSTRACT: Plant residues and certain fertilizers accelerate soil acidification and increase the levels of aluminum-Al +3 in soils under no-tillage (NT). Silicates act as acidity amendments and as a source of silicon as in $\mathrm{H}_{4} \mathrm{SiO}_{4}$. An increase in the $\mathrm{pH}$ of soil solution causes the deprotonation of $\mathrm{H}_{4} \mathrm{SiO}_{4}$ and generates the anionic form $\left(\mathrm{H}_{3} \mathrm{SiO}_{4}^{-}\right)$. The aim of this study was to evaluate the ionic speciation of $\mathrm{Si}, \mathrm{Al}, \mathrm{Ca}, \mathrm{Mg}$ and $\mathrm{K}$ in aqueous extracts by means of a software calculation. Since 2006, a field experiment has been under way on an Oxisol under NT subjected to lime and calcium-magnesium silicate applications under four crop systems. The amendments were applied in Oct 2006 and in Oct 2011, aiming to raise base saturation to $70 \%$. Soil samples were collected in Oct 2013, at depths of 0-5, 5-10, 10-20, 20-40 and 40-60 cm. Both $\mathrm{Ca}$ and $\mathrm{Mg}$ formed complexes with dissolved organic carbon (DOC) whereas the same was not observed for potassium. These three basic cations were mostly in their free forms regardless of treatment, while Al was mostly complexed with DOC even at the lowest depths $(40-60 \mathrm{~cm})$. The highest value of free $\mathrm{Al}$ form was $15 \%$. Si was almost $100 \%$ as $\mathrm{H}_{4} \mathrm{SiO}_{4}$, and its activity was similar to its concentration in solution for all crop systems and at all depths, regardless of amendment applied. The percentages of $\mathrm{H}_{3} \mathrm{SiO}_{4}{ }^{-}$and $\mathrm{Al}_{3} \mathrm{H}_{3} \mathrm{SiO}_{4}{ }^{2+}$ were irrelevant, providing more phytoavailable $\mathrm{H}_{4} \mathrm{SiO}_{4}$ in soil solution.

Keywords: soil solution, cations speciation, aqueous extraction, amendments

\section{Introduction}

Plants absorb silicon ( $\mathrm{Si}$ ) in the form of silicic acid $\left(\mathrm{H}_{4} \mathrm{SiO}_{4}\right)$. It is gradually becoming recognized nowadays as an important element for plants (Datnoff et al., 2007). Tropical soils are generally deficient in this element, and soluble sources of $\mathrm{Si}$ are added to highly weathered soils with low Si available content, less than $2 \mathrm{mg} \mathrm{kg}^{-1}$ (Fox and Silva, 1978), and this practice contributes to improvements in the nutritional status of plants, and promotes resistance to a variety of fungal diseases (Datnoff et al., 2001). On the other hand, Si can be strongly bound to the mineral phases and, therefore, becomes less available for plants (Hobara et al., 2016). Calcium-magnesium silicates $(\mathrm{Ca} /$ $\mathrm{MgSiO}_{3}$ ) correct acidity in the soil by raising $\mathrm{pH}$ values and provide readily available silicon, calcium and magnesium to plants through the following reaction: $\mathrm{Ca} / \mathrm{MgSiO}_{3}+\mathrm{H}_{2} \mathrm{O} \rightarrow \mathrm{Ca}^{2+} / \mathrm{Mg}^{2+}+\mathrm{SiO}_{4}{ }_{4-}^{4-}$ (Ramos et al., 2006). In the case of $\mathrm{Si}$, silicates dissolve in acidic soil and promote the release of $\mathrm{H}_{4} \mathrm{SiO}_{4}$ in solution (equations $1,2,3$ and 4 of chemical equilibrium), with silicon in a form available to plants (Jones and Handreck, 1967). Levels of $\mathrm{Ca}, \mathrm{Mg}$ and $\mathrm{K}$ increase with increases in the soil $\mathrm{pH}$, which is also a consequence of silicate fertilization (Ramos et al., 2006).

$$
\begin{aligned}
& \mathrm{SiO}_{4}{ }^{4-}+\mathrm{H}_{2} \mathrm{O}_{\text {(soil) }} \rightarrow \mathrm{HSiO}_{4}{ }^{3-}+\mathrm{OH}^{-} \log K^{0}=-45.95 \quad \text { Eq. } 1 \\
& \mathrm{HSiO}_{4}{ }^{3-}+\mathrm{H}_{2} \mathrm{O}_{\text {(soil) }} \rightarrow \mathrm{H}_{2} \mathrm{SiO}_{4}{ }^{2-}+\mathrm{OH}^{-} \log K^{0}=-32.85 \text { Eq. } 2 \\
& \mathrm{H}_{2} \mathrm{SiO}_{4}{ }^{2-}+\mathrm{H}_{2} \mathrm{O}_{\text {(soill }} \rightarrow \mathrm{H}_{3} \mathrm{SiO}_{4}{ }^{-}+\mathrm{OH}^{-} \log K^{0}=-22.98 \text { Eq. } 3 \\
& \mathrm{H}_{3} \mathrm{SiO}_{4}{ }^{-}+\mathrm{H}_{2} \mathrm{O}_{\text {(soil) }} \rightarrow \mathrm{H}_{4} \mathrm{SiO}_{4}+\mathrm{OH}^{-} \log K^{0}=-9.71 \quad \text { Eq. } 4
\end{aligned}
$$

The NT system can change the available Si content in soil solution and minimize the harmful effects of $\mathrm{Al}^{3+}$, especially in the surface layers, where there is greater concentration of organic matter $(\mathrm{OM})$. Much of the $\mathrm{Al}$ is present in the soil solid phase and is in equilibrium with the liquid phase (Hiradate, 2011). The greatest contribution of OM in soils under no-tillage (NT) systems minimizes the toxic effects of $\mathrm{Al}$ through the strong link of this metal with anions derived from organic acids. In this sense, silicate application under NT, in addition to providing available $\mathrm{Si}$, has the potential to make $\mathrm{Si}$ even more available due to the strong interaction of organic matter components with aluminum, which is even stronger than hydroxyl ions $\left(\mathrm{OH}^{-}\right)$, and then provides more $\mathrm{Si}$ in soil solution by reducing the formation of aluminosilicates. Chemical (or ionic) speciation provides a detailing of the chemical composition of soil solution, including the forms of elements in the solution. $\mathrm{Ca}, \mathrm{Mg}$, $\mathrm{K}$ and $\mathrm{Si}$ species found in soil solution under NT, mainly in the surface layers $(0-10 \mathrm{~cm})$, or even more in soils subjected to the application of amendments, are still little known. Thus, detailed information about the species is necessary because soil management is able to alter them and, thus, interfere in their availability to plants.

Seeking to unite the benefits of surface application of amendments with NT, our aim was to evaluate the ionic speciation of $\mathrm{Si}, \mathrm{Al}$ and exchangeable cations $(\mathrm{Ca}, \mathrm{Mg}$ and $\mathrm{K}$ ) at five sampling depths, considering a possible residual effect of both practices, for four crop systems, assuming that (i) $\mathrm{H}_{4} \mathrm{SiO}_{4}$ is the predominant species of soluble $\mathrm{Si}$ in tropical soils and suffers no interference from the application of lime and/or calcium-magnesium silicate; (ii) the dissociated form of $\mathrm{H}_{4} \mathrm{SiO}_{4}\left(\mathrm{H}_{3} \mathrm{SiO}_{4}{ }^{-}\right)$is irrelevant to the complexation of aluminum in soil solution due to the 
maximum $\mathrm{pH}$ value reached by the application of soil amendments being insufficient to provide a good amount of the anion silicate; and that (iii) organic ligands bear, in the main, responsibility for $\mathrm{Al}, \mathrm{Ca}$ and $\mathrm{Mg}$ complexation in soils under NT even at lower depths and after the application of soil amendments.

\section{Materials and Methods}

\section{Description of the area and soil characterization}

The experimental area has been cultivated under NT since 2002, and this experiment has been under way since 2006 in Botucatu, in the state of São Paulo, Brazil, (48 23' W; 22 51' S; $765 \mathrm{~m}$ a.s.l). The soil is a typical clayey Hapludox, and chemical analysis results prior to the setting-up of the experiment were obtained as Raij (1998) (Table 1). According to the Köppen classification, the prevailing climate in the region is Cwa (high altitude, tropical climate with dry winters and hot rainy summers).

\section{Design of the experiment and description of the treatment}

The design of the experiment was a randomized block with split plots. The plots consisted of four crop systems (I - Season - Forage; II - Season - Off-season; III - Season - Fallow; IV - Season - Green-manure) and subplots comprising two sources of lime (limestone and calcium-magnesium silicates) and a control (without corrective), with four replications ( $n=4)$. This design was adopted so as to enable the experiment to be conducted in a fully mechanized way. Soil samples were collected by using a probe at the following depths: $0-5,-5-10$, $-10-20,-20-40$ and $-40-60 \mathrm{~cm}$. Although the NT system provides greater changes in the upper soil layers, we further planned to evaluate soil solution speciation down to a depth of $60 \mathrm{~cm}$ because the root systems of certain grasses cultivated in the experiment since 2006 have the potential to grow down to lower depths, which contributes to a higher content of soil organic matter (SOM) at lower depths. The organic acids excreted may contribute significantly to the contents of DOC. In order to correct the soil acidity, dolomitic lime (Effective $\mathrm{CaCO}_{3}$ equivalent $-\mathrm{ECCE}=90 \%, \mathrm{CaO}=36 \%$ and $\mathrm{MgO}=12 \%$ ) and calcium-magnesium silicate $(\mathrm{ECCE}=80 \%=\mathrm{CaO} 34 \%$, $\mathrm{MgO}=10 \%$ and $\mathrm{SiO}_{2}=22 \%$ ) were used: doses were applied in Oct, 2006 (3.8 and $4.1 \mathrm{Mg} \mathrm{ha}^{-1}$, respectively), followed by a reapplication in Oct, 2011 (4.7 and $5.3 \mathrm{Mg}$ $\mathrm{ha}^{-1}$, respectively), aiming to raise base saturation (V\%)

Table 1 - Chemical properties of Oxisol (2006).

\begin{tabular}{|c|c|c|c|c|c|c|c|c|c|c|}
\hline Depth & $\mathrm{pH}$ & $\mathrm{OM}$ & $P$ & $\mathrm{Si}$ & $\mathrm{Ca}$ & $\mathrm{Mg}$ & K & Al & $\mathrm{H}+\mathrm{Al}$ & V \\
\hline $\mathrm{cm}$ & $0.01 \mathrm{M} \mathrm{CaCl}_{2}$ & $\mathrm{~g} \mathrm{dm}^{-3}$ & $\mathrm{mg}$ & $\mathrm{dm}^{-3}$ & 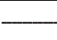 & $-\mathrm{mr}$ & $\mathrm{nol}_{\mathrm{c}} \mathrm{d}$ & $m^{-3}-$ & - & $\%$ \\
\hline $0-5$ & 4.7 & 23 & 8.7 & 7.5 & 21 & 9 & 2.1 & 4 & 50.3 & 27 \\
\hline $5-10$ & 4.4 & 19 & 7.9 & 6.3 & 11 & 6 & 1.1 & 6 & 69.3 & 25 \\
\hline $10-20$ & 4.0 & 18 & 2.0 & 6.2 & 10 & 4 & 0.6 & 5 & 61.2 & 24 \\
\hline $20-40$ & 4.0 & 17 & 3.0 & 6.0 & 12 & 4 & 0.7 & 6 & 64.3 & 24 \\
\hline $40-60$ & 4.0 & 15 & 2.1 & 6.7 & 9 & 4 & 0.6 & 12 & 80.1 & 22 \\
\hline
\end{tabular}

to $70 \%$. In the reapplication the characteristics of lime were: $\mathrm{ECCE}=74 \%, \mathrm{CaO}=35 \%$ and $\mathrm{MgO}=12 \%$, and the characteristics of silicate were: $\mathrm{ECCE}=66 \%, \mathrm{CaO}$ $=32 \%, \mathrm{MgO}=9 \%$ and $\mathrm{SiO}_{2}=22 \%$.

The following crops: soybean (Glycine max), corn (Zea mays), rice (Oryza sativa), soybean, corn, bean (Phaseolus vulgaris) and rice (sown in Nov), comprised the seasons' crops in the following agricultural years: 2006/07, 2007/08, 2008/09, 2009/10, 2010/11, 2011/12 and 2012/13, respectively.

Soil samples were air-dried, slightly crushed, passed through a $2 \mathrm{~mm}$ sieve and then stored in plastic bags kept in the shade at room temperature until analysis.

\section{Extraction and chemical analysis of soil solution}

The aqueous extract method was used to extract the soil solution using a 1:1 soil:water ratio (Wolt, 1994). Twenty grams of soil and $20 \mathrm{~mL}$ of ultrapure water were placed in a $50 \mathrm{~mL}$ centrifuge tube, which was then placed in a horizontal table shaker for $15 \mathrm{~min}$ at 150 rpm, followed by $1 \mathrm{~h}$ rest. The solution was then shaken again for $5 \mathrm{~min}$ followed by centrifugation for $30 \mathrm{~min}$ at $1,844 \times \mathrm{g}$. Three extractions were performed to obtain extracts of cations, anions, and dissolved organic carbon, which were determined separately. To obtain the extract for determining cations and anions, the solution was filtered through a $0.45 \mu \mathrm{m}$ cellulose membrane and stored for 15 days in a refrigerator until readings were taken. The extract for determining DOC (dissolved organic carbon) was filtered through a $0.7 \mu \mathrm{m} \mathrm{GF} / \mathrm{F}$ glass microfiber filter that was previously calcinated at $500{ }^{\circ} \mathrm{C}$ for 6 $\mathrm{h}$ and then packed in glass vials subjected to washing and decontamination in hydrochloric acid solution $120 \%$ $\mathrm{HCl}$ ). About $0.1 \mathrm{~mL}$ of $30 \mathrm{mmol} \mathrm{L}^{-1} \mathrm{HgCl}_{2}$ solution was used for this last extraction with the aim of avoiding the proliferation of microrganisms. The filtered extract was stored for 30 days in a freezer until readings were taken.

All filtering for obtaining the extracts were performed with the aid of $25 \mathrm{~mm}$ diameter filters for the fraction of cations, anions and DOC. These supports were coupled in $60 \mathrm{~mL}$ plastic syringes in which the extract obtained after centrifugation was placed, and filtered under pressure by manual force. In the solutions, immediately after centrifugation, $\mathrm{pH}$ was determined by potentiometry and the electrical conductivity (EC) by electrometry (used to calculate the ionic strength of the soil solution). The cation contents $(\mathrm{K}, \mathrm{Na}, \mathrm{Ca}, \mathrm{Mg}, \mathrm{Cu}$, $\mathrm{Zn}, \mathrm{Fe}, \mathrm{Al}$ and $\mathrm{Si}$ ) were determined by ICP-OES, whereas the anion contents $\left(\mathrm{NO}_{2}{ }^{-}, \mathrm{NO}_{3}{ }^{-}, \mathrm{SO}_{4}{ }^{2-}, \mathrm{PO}_{4}{ }^{3-}, \mathrm{Cl}^{-}\right.$and $\mathrm{F}^{-}$, were determined by ionic chromatography; and the DOC, in carbon analyzer. Ultrapure water was used as control, and reference standards were used as quality control and for calibration curves of the equipment.

The ionic force of solution $(I)$ was obtained according to equation 5 (calculated automatically by the Visual MINTEQ software program), cited in Sposito (1989), taking into account the contents of certain elements in the solution. 
$I=1 / 2 \sum_{i=1}^{n} C i \cdot(Z i)^{2}$

$\mathrm{Ci}$ is the concentration $\left(\mathrm{mol} \mathrm{L}^{-1}\right)$ of each ion from solution and $Z i$ its charge.

\section{Ionic speciation of soil solution}

The ionic speciation of the soil solution was based on the values of the concentrations of organic and inorganic cations and anions, by using the Visual MINTEQ software program (Gustafsson, 2013). The free species, the complexed species and the activity of the elements in solution were then estimated. The species linked to organic anions were estimated based on the DOC content by "Gaussian DOM" model (Grimm et al., 1991). The software has a database containing the values of the constant values of formation and stability of all possible species. Thus, it was possible to simulate the percentage distribution of species formed at a given $\mathrm{pH}$, measured in the aqueous extract. All determinations for a given sample were inserted into the software program, and then the speciation was calculated. The concentration of each element in each aqueous extract was verified for each sample. For this, all species for a given element were summed, and then this value was verified against the determined value (from the extract). The average (n $=4$ ) has been used for the species calculated in each sample.

\section{Statistical analysis}

For the statistical analysis, the results were plotted as a percentage distribution of species followed by mean standard deviations for lime and Ca-Mg silicate, separately, and for each crop system, aiming to verify the isolated effect of the soil amendments over the main species in question. The distribution in percentages of each species in the soil solution was obtained as a function of the soil amendments application and sampling depths, for each crop system. Next, the percentages of each species were compared at each depth and each crop system using the mean standard deviation (with four replications). Pearson correlation and linear regression analyses were configured by the Excel software program and extracted between the total concentrations of the ions and the activities of their free forms in aqueous solution. Linear regressions were constructed with $95 \%$ of confidence, which is in alignment with the probability of error in the fit of the regression model obtained by the Excel software program.

\section{Results and Discussion}

\section{Aluminum}

Regardless of correctives and crop systems, the activity of aluminum in free form $\left(a \mathrm{Al}^{3+}\right)$ was positively correlated with the total metal concentration in aqueous extract $(\mathrm{Al} t)$ in the first three layers (from 0-5; $-5-10$ and $-10-20 \mathrm{~cm})$. On the other hand, there was no correlation between the samples from the remaining two layers (20-40 and 40-60 cm) (Table 2 and Figure 1). Ionic aluminum in free form $\left(\mathrm{Al}^{3+}\right)$ is potentially the most phytotoxic form of this metal (Ma and Furu-

Table 2 - Coefficients of determination and Pearson correlation between total concentrations of aluminum, calcium, magnesium and potassium and their respective activities of their free forms in solution $\left(\mathrm{Al}^{3+}, \mathrm{Ca}^{2+}, \mathrm{Mg}^{2+}\right.$ and $\left.\mathrm{K}^{+}\right)$, and silicon and silicic acid activity $\left(\mathrm{H}_{4} \mathrm{SiO}_{4}\right)$, in aqueous extracts for different layers of an Oxisol under no-tillage $(p<0.05)$.

\begin{tabular}{lrrrrc}
\hline Depth (cm) & Al & \multicolumn{1}{c}{ Ca } & Mg & $\mathrm{K}$ & $\mathrm{Si}$ \\
\hline 0-5 & $\mathrm{R}^{2}=0.68$ & $\mathrm{R}^{2}=0.98$ & $\mathrm{R}^{2}=0.99$ & $\mathrm{R}^{2}=0.99$ & $\mathrm{R}^{2}=0.99$ \\
& $r=0.83$ & $r=0.99$ & $r=0.99$ & $r=0.99$ & $r=0.99$ \\
stdev & $4 \times 10^{-6}$ & $2 \times 10^{-5}$ & $6 \times 10^{-6}$ & $5 \times 10^{-6}$ & $5 \times 10^{-6}$ \\
\hline \multirow{2}{*}{-10 } & $\mathrm{R}^{2}=0.70$ & $\mathrm{R}^{2}=0.91$ & $\mathrm{R}^{2}=0.99$ & $\mathrm{R}^{2}=0.99$ & $\mathrm{R}^{2}=1$ \\
& $r=0.84$ & $r=0.95$ & $r=0.99$ & $r=0.99$ & $r=1$ \\
stdev & $9 \times 10^{-7}$ & $2 \times 10^{-5}$ & $2 \times 10^{-6}$ & $9 \times 10^{-7}$ & $2 \times 10^{-7}$ \\
\hline \multirow{2}{*}{ 10-20 } & $\mathrm{R}^{2}=0.74$ & $\mathrm{R}^{2}=0.98$ & $\mathrm{R}^{2}=0.99$ & $\mathrm{R}^{2}=0.99$ & $\mathrm{R}^{2}=1$ \\
& $r=0.86$ & $r=0.99$ & $r=0.99$ & $r=0.99$ & $r=1$ \\
stdev & $2 \times 10^{-7}$ & $4 \times 10^{-6}$ & $1 \times 10^{-6}$ & $5 \times 10^{-7}$ & $2 \times 10^{-8}$ \\
\hline \multirow{2}{*}{ 20 - 40 } & $\mathrm{R}^{2}=0.32$ & $\mathrm{R}^{2}=0.94$ & $\mathrm{R}^{2}=0.99$ & $\mathrm{R}^{2}=0.99$ & $\mathrm{R}^{2}=0.99$ \\
& $r=0.56$ & $r=0.97$ & $r=0.99$ & $r=0.99$ & $r=0.99$ \\
stdev & $2 \times 10^{-7}$ & $2 \times 10^{-5}$ & $2 \times 10^{-6}$ & $4 \times 10^{-7}$ & $4 \times 10^{-7}$ \\
\hline \multirow{2}{*}{ 40 - 60 } & $\mathrm{R}^{2}=0.11$ & $\mathrm{R}^{2}=0.95$ & $\mathrm{R}^{2}=0.99$ & $\mathrm{R}^{2}=0.99$ & $\mathrm{R}^{2}=1$ \\
& $r=0.33$ & $r=0.97$ & $r=0.99$ & $r=0.99$ & $r=1$ \\
stdev & $9 \times 10^{-7}$ & $1 \times 10^{-5}$ & $2 \times 10^{-6}$ & $3 \times 10^{-7}$ & $3 \times 10^{-8}$ \\
\hline
\end{tabular}
stdev $=$ Standard deviation.
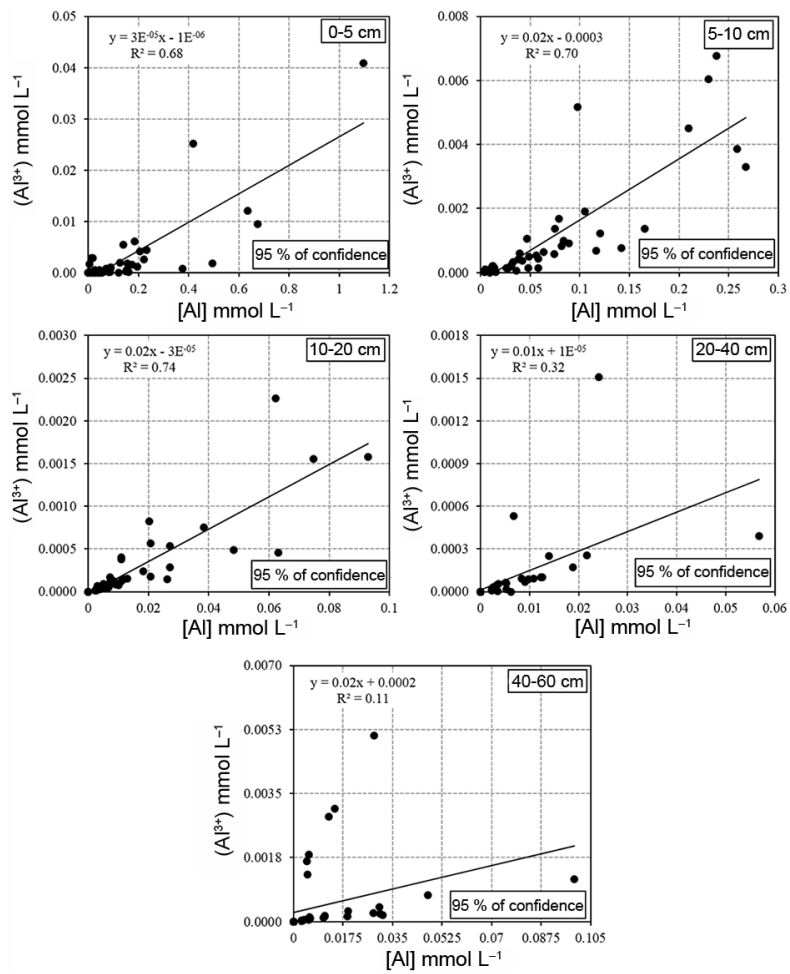

Figure 1 - Linear regressions for aluminum concentration ([AI]) and the activity of its free form ((All $\left.\left.{ }^{3+}\right)\right)$ in solution at five depths of an Oxisol under NT. 
kawa, 2003). The differences found between Alt and $a \mathrm{Al}^{3+}$ in aqueous extract suggest that the total concentration of aluminum represents neither its availability nor its potential phytotoxicity (Zambrosi et al., 2007), which is attributable to free-form $\mathrm{Al}^{3+}$. Total metal concentrations, $\mathrm{pH}$ values and the levels of $I$ in soil solution in the aqueous extract are shown in Tables $3,4,5$ and 6 .

Table 3 - Acidity correctives, pH values, dissolved organic carbon (DOC), inorganic anions, cations and ionic strength (I), micronutrients, sodium, nitrite, phosphate and sulfur on soil solution in five depths of an Oxisol under no-tillage, in Season - Forage system. QL = quantification limit, $\mathrm{mmol}^{-1}$.

\begin{tabular}{|c|c|c|c|c|c|c|c|c|}
\hline \multicolumn{9}{|c|}{ Season - Forage } \\
\hline \multicolumn{2}{|c|}{ Treatment Depth } & $\mathrm{pH}$ & DOC & $\mathrm{N}-\mathrm{NO}_{3}^{-}$ & $\mathrm{Cl}^{-}$ & $\mathrm{S} \mathrm{SO}_{4}{ }^{2-}$ & $\mathrm{P}$ & $\mathrm{F}^{-}$ \\
\hline \multicolumn{3}{|c|}{$\mathrm{cm}$} & & & & $\mathrm{mol} \mathrm{L}^{-1}$ & & \\
\hline Control & \multirow{3}{*}{$0-5$} & $4.7 \pm(0.0)$ & $5.5 \pm(0.7)$ & $0.43 \pm(0.18)$ & $0.15 \pm(0.13)$ & $0.091 \pm(0.011)$ & $0.014 \pm(0.006)$ & $0.009 \pm(0.001)$ \\
\hline Silicate & & $6.1 \pm(0.1)$ & $6.1 \pm(0.4)$ & $0.59 \pm(0.23)$ & $0.10 \pm(0.02)$ & $0.120 \pm(0.047)$ & $0.011 \pm(0.003)$ & $0.042 \pm(0.017)$ \\
\hline Lime & & $5.7 \pm(0.1)$ & $8.0 \pm(1.4)$ & $0.60 \pm(0.30)$ & $0.08 \pm(0.03)$ & $0.104 \pm(0.019)$ & $0.016 \pm(0.010)$ & $0.010 \pm(0.001)$ \\
\hline Control & \multirow{3}{*}{$5-10$} & $4.6 \pm(0.1)$ & $3.0 \pm(0.3)$ & $0.29 \pm(0.07)$ & $0.11 \pm(0.07)$ & $0.274 \pm(0.317)$ & $0.006 \pm(0.001)$ & $0.009 \pm(0.001)$ \\
\hline Silicate & & $5.3 \pm(0.1)$ & $3.7 \pm(0.2)$ & $0.42 \pm(0.16)$ & $0.10 \pm(0.02)$ & $0.222 \pm(0.062)$ & $0.009 \pm(0.003)$ & $0.012 \pm(0.003)$ \\
\hline Lime & & $5.1 \pm(0.0)$ & $5.3 \pm(0.0)$ & $0.30 \pm(0.09)$ & $0.09 \pm(0.02)$ & $0.149 \pm(0.023)$ & $0.012 \pm(0.005)$ & $0.010 \pm(0.000)$ \\
\hline Control & \multirow{3}{*}{$10-20$} & $4.7 \pm(0.1)$ & $1.9 \pm(0.6)$ & $0.30 \pm(0.04)$ & $0.11 \pm(0.06)$ & $0.061 \pm(0.011)$ & $0.004 \pm(0.000)$ & $0.008 \pm(0.002)$ \\
\hline Silicate & & $4.8 \pm(0.1)$ & $1.9 \pm(0.5)$ & $0.55 \pm(0.37)$ & $0.10 \pm(0.02)$ & $0.137 \pm(0.039)$ & $0.003 \pm(0.000)$ & $0.009 \pm(0.001)$ \\
\hline Lime & & $4.9 \pm(0.0)$ & $2.9 \pm(0.6)$ & $0.23 \pm(0.08)$ & $0.07 \pm(0.03)$ & $0.099 \pm(0.023)$ & $0.005 \pm(0.001)$ & $0.008 \pm(0.000)$ \\
\hline Control & \multirow{3}{*}{$20-40$} & $4.8 \pm(0.1)$ & $5.2 \pm(0.3)$ & $0.27 \pm(0.07)$ & $0.09 \pm(0.03)$ & $0.105 \pm(0.022)$ & $13.032 \pm(19.538)$ & $\mathrm{nd}^{*}$ \\
\hline Silicate & & $4.8 \pm(0.0)$ & $5.3 \pm(0.5)$ & $0.29 \pm(0.11)$ & $0.13 \pm(0.03)$ & $0.127 \pm(0.027)$ & $0.009 \pm(0.004)$ & $0.022 \pm(0.000)$ \\
\hline Lime & & $4.9 \pm(0.0)$ & $5.6 \pm(0.5)$ & $0.23 \pm(0.07)$ & $0.10 \pm(0.02)$ & $0.129 \pm(0.014)$ & $0.009 \pm 10.0$ & $0.008 \pm 10$. \\
\hline Control & \multirow{3}{*}{$40-60$} & $4.7 \pm(0.3)$ & $3.8 \pm(0.4)$ & $0.19 \pm(0.04)$ & $0.07 \pm(0.03)$ & $0.145 \pm(0.056)$ & $0.005 \pm 10.0$ & $0.010 \pm 10.0$ \\
\hline Silicate & & $4.9 \pm(0.0)$ & $3.6 \pm(0.5)$ & $0.20 \pm(0.05)$ & $0.07 \pm(0.01)$ & $0.146 \pm(0.037)$ & $0.008 \pm(0.004)$ & $0.010 \pm(0.000)$ \\
\hline Lime & & $4.9 \pm(0.0)$ & $3.6 \pm(0.4)$ & $0.20 \pm(0.08)$ & $0.08 \pm(0.01)$ & $0.133 \pm(0.017)$ & $0.005 \pm(0.002)$ & $\mathrm{nd}^{*}$ \\
\hline \multicolumn{2}{|c|}{ Treatment Depth } & $\mathrm{Al}$ & $\mathrm{Ca}$ & $\mathrm{Mg}$ & $\mathrm{K}$ & $\mathrm{Si}$ & $\mathrm{Fe}$ & 1 \\
\hline & \multirow[t]{2}{*}{$\mathrm{cm}$} & & & & & & & \\
\hline Control & & $0.17 \pm(0.12)$ & $0.10 \pm(0.03)$ & $0.12 \pm(0.04)$ & $0.39 \pm 10.3$ & $0.14 \pm(0.06)$ & $0.020 \pm(0.010)$ & $1.32 \pm(0.42)$ \\
\hline Silicate & \multirow[t]{2}{*}{$0-5$} & $0.10 \pm(0.06)$ & $0.33 \pm(0.07)$ & $0.21 \pm 0$ & $0.36 \pm(0.09)$ & $0.10 \pm($ & & \\
\hline Lime & & $0.39 \pm(0.26)$ & $0.25 \pm(0.06)$ & $0.21 \pm(0.04)$ & $0.32 \pm(0.09)$ & $0.36 \pm 10$ & $0.056 \pm 1$ & $1.89 \pm$ \\
\hline Control & \multirow{3}{*}{$5-10$} & $0.03 \pm(0.03)$ & $0.14 \pm(0.06)$ & $0.10 \pm(0.04)$ & $0.21 \pm(0.14)$ & $0.06 \pm(0.04)$ & $0.005 \pm 10$. & $1.02 \pm($ \\
\hline Silicate & & $0.07 \pm(0.01)$ & $0.24 \pm(0.03)$ & $0.16 \pm(0.03)$ & $0.17 \pm(0.03)$ & $0.07 \pm(0.02)$ & $0.012 \pm 10$. & 3) \\
\hline Lime & & $0.07 \pm(0.05)$ & $0.17 \pm(0.03)$ & $0.13 \pm 10$ & $0.18 \pm(0.07)$ & $0.08 \pm 10$ & 0.0 & $1.13 \pm$ \\
\hline Control & \multirow{3}{*}{$10-20$} & $0.01 \pm(0.00)$ & $0.09 \pm(0.02)$ & $0.06 \pm(0.01)$ & $0.09 \pm(0.04)$ & $0.03 \pm(0.02)$ & 0.0 & $0.72 \pm 1$ \\
\hline Silicate & & $0.01 \pm(0.01)$ & $0.17 \pm(0.04)$ & $0.12 \pm(0.03)$ & $0.010 \pm(0.04)$ & $0.03 \pm(0.02)$ & $0.002 \pm 0$. & $1.20 \pm($ \\
\hline Lime & & $0.01 \pm(0.01)$ & $0.11 \pm(0.02)$ & $0.08 \pm(0.01)$ & $0.12 \pm(0.06)$ & $0.03 \pm(0.01)$ & $0.003 \pm(0.002)$ & $0.82 \pm 10$ \\
\hline Control & \multirow{3}{*}{$20-40$} & $0.01 \pm(0.00)$ & $0.14 \pm(0.05)$ & $0.09 \pm(0.02)$ & $11 \pm(0.01)$ & $0.04 \pm 10$ & $0.001 \pm 10$ & $280.01 \pm(4$ \\
\hline Silicate & & $0.01 \pm(0.00)$ & $0.15 \pm(0.06)$ & $0.09 \pm(0.03)$ & $0.07 \pm(0.02)$ & $0.05 \pm(0.04)$ & $0.002 \pm 10$ & $0.95 \pm 1($ \\
\hline Lime & & $0.01 \pm(0.00)$ & $0.15 \pm(0.05)$ & $0.10 \pm(0.02)$ & $0.09 \pm(0.04)$ & $0.03 \pm(0.02)$ & $0.001 \pm 10.0$ & $0.94 \pm 1 C$ \\
\hline Control & \multirow{3}{*}{$40-60$} & $0.01 \pm(0.01)$ & $0.10 \pm(0.06)$ & $0.06 \pm(0.03)$ & $0.05 \pm(0.04)$ & $0.04 \pm(0.03)$ & $0.004 \pm(0.004)$ & $0.65 \pm(0.23)$ \\
\hline Silicate & & $0.03 \pm(0.01)$ & $0.15 \pm(0.06)$ & $0.08 \pm(0.02)$ & $0.05 \pm(0.02)$ & $0.04 \pm(0.03)$ & $0.005 \pm(0.006)$ & $0.87 \pm(0.18)$ \\
\hline Lime & & $0.02 \pm(0.01)$ & $0.14 \pm(0.06)$ & $0.08 \pm(0.02)$ & $0.05 \pm(0.02)$ & $0.03 \pm(0.02)$ & $0.003 \pm(0.003)$ & $0.88 \pm(0.22)$ \\
\hline \multicolumn{2}{|c|}{ Treatment Depth } & $\mathrm{Mn}$ & $\mathrm{Cu}$ & $\mathrm{Zn}$ & $\mathrm{Na}$ & ${\mathrm{N}-\mathrm{NO}_{2}^{-}}^{-}$ & $\mathrm{P}^{-} \mathrm{PO}_{4}^{3-}$ & $S$ \\
\hline & \multirow[t]{2}{*}{$\mathrm{cm}$} & & & & & & & \\
\hline Control & & $0.018 \pm(0.008)$ & & & $0.06 \pm(0.05)$ & $\mathrm{nd}^{*}$ & & $0.135 \pm(0.041)$ \\
\hline Silicate & \multirow[t]{2}{*}{$0-5$} & $0.001 \pm(0.001)$ & $0.0004 \pm(0.0001)$ & $0.0001 \pm(0.0001)$ & $0.05 \pm(0.04)$ & $0.056 \pm(0.038)$ & $0.0036 \pm(0.0009)$ & $0.187 \pm(0.041)$ \\
\hline Lime & & $0.003 \pm(0.002)$ & $0.0004 \pm(0.0001)$ & $0.0001 \pm(0.0001)$ & $0.04 \pm(0.03)$ & $0.008 \pm(0.005)$ & $0.0053 \pm(0.0031)$ & $0.138 \pm(0.051)$ \\
\hline Control & \multirow{3}{*}{$5-10$} & $0.018 \pm(0.008)$ & $0.0001 \pm(0.0000)$ & $0.0005 \pm(0.0002)$ & $0.10 \pm(0.06)$ & $\mathrm{nd}^{*}$ & $0.0020 \pm(0.0002)$ & $0.089 \pm(0.016)$ \\
\hline Silicate & & $0.003 \pm(0.002)$ & $0.0002 \pm(0.0000)$ & $0.0003 \pm(0.0001)$ & $0.05 \pm(0.04)$ & $0.005 \pm(0.002)$ & $0.0031 \pm(0.0009)$ & $0.255 \pm 10$. \\
\hline Lime & & $0.006 \pm(0.001)$ & $0.0002 \pm(0.0000)$ & $0.0005 \pm(0.0002)$ & $0.03 \pm(0.03)$ & $0.007 \pm(0.005)$ & $0.0038 \pm(0.0018)$ & $0.146 \pm(0.041)$ \\
\hline Control & & $0.011 \pm(0.005)$ & $0.0001 \pm(0.0000)$ & $0.0004 \pm(0.0001)$ & $0.06 \pm(0.07)$ & $\mathrm{nd}^{*}$ & $0.0012 \pm(0.0000)$ & $0.062 \pm(0.011)$ \\
\hline Silicate & $10-20$ & $0.007 \pm(0.001)$ & $0.0001 \pm(0.0000)$ & $0.0003 \pm(0.0001)$ & $0.05 \pm(0.03)$ & $\mathrm{nd}^{*}$ & $0.0011 \pm(0.0000)$ & $0.129 \pm(0.022)$ \\
\hline Lime & & $0.010 \pm(0.004)$ & $0.0001 \pm(0.0000)$ & $0.0002 \pm(0.0001)$ & $0.04 \pm(0.03)$ & $0.002 \pm(0.000)$ & $0.0016 \pm(0.0003)$ & $0.114 \pm(0.018)$ \\
\hline Control & & $17.751 \pm(26.601)$ & $0.2076 \pm(0.2767)$ & $0.3717 \pm(0.5573)$ & $0.07 \pm(0.05)$ & $0.004 \pm(0.002)$ & $4.2502 \pm(6.3721)$ & $142.526 \pm(213.627)$ \\
\hline Silicate & $20-40$ & $0.018 \pm(0.007)$ & $0.0001 \pm(0.0000)$ & $0.0001 \pm(0.0000)$ & $0.06 \pm(0.04)$ & $0.002 \pm(0.000)$ & $0.0029 \pm(0.0012)$ & $0.121 \pm(0.037)$ \\
\hline Lime & & $0.012 \pm(0.004)$ & $0.0000 \pm(0.0000)$ & $0.0001 \pm(0.0001)$ & $0.06 \pm(0.03)$ & $0.002 \pm(0.000)$ & $0.0028 \pm(0.0013)$ & $0.151 \pm(0.028)$ \\
\hline Control & & $0.004 \pm(0.002)$ & $0.0000 \pm(0.0000)$ & $0.0001 \pm(0.0001)$ & $0.07 \pm(0.05)$ & $0.005 \pm(0.000)$ & $0.0016 \pm(0.0005)$ & $0.099 \pm(0.038)$ \\
\hline Silicate & $40-60$ & $0.010 \pm(0.007)$ & $0.0000 \pm(0.0000)$ & $0.0001 \pm(0.0000)$ & $0.06 \pm(0.03)$ & $0.003 \pm(0.000)$ & $0.0026 \pm(0.0012)$ & $0.135 \pm(0.056)$ \\
\hline Lime & & $0.005 \pm(0.002)$ & $0.0000 \pm(0.0000)$ & $0.0001 \pm(0.0000)$ & $0.07 \pm(0.04)$ & $\mathrm{nd}^{*}$ & $0.0018 \pm(0.0006)$ & $0.139 \pm(0.025)$ \\
\hline
\end{tabular}

Values between parentheses mean the average deviation $(n=4) ; n d^{*}=$ not detected: $Q \mathrm{~L}: \mathrm{F}^{-}=0.0026 ; \mathrm{N}^{-} \mathrm{NO}_{2}{ }^{-}=0.0011$. 
On average, $a \mathrm{Al}^{3+}$ corresponded to approximately $3 \%$ of the total aluminum concentration in all five soil layers for all treatments studied. Zambrosi et al. (2007) found differences of almost $1 \%$ in their study compared to $40 \%$ in other authors' studies for $a \mathrm{Al}^{3+}$ in relation to Alt, respectively. However, these other authors did not determine the fluoride content $\left(\mathrm{F}^{-}\right)$for calculating the activity of aluminum in solution. Fluoride ions $\left(\mathrm{F}^{-}\right.$an-

Table 4 - Acidity correctives, pH values, dissolved organic carbon (DOC), inorganic anions, cations and ionic strength (I), micronutrients, sodium, nitrite, phosphate and sulfur on soil solution in five depths of an Oxisol under no-tillage, in Season - Off-season system. QL $=$ quantification limit, $\mathrm{mmol} \mathrm{L}^{-1}$.

\begin{tabular}{|c|c|c|c|c|c|c|c|c|}
\hline \multicolumn{9}{|c|}{ Season - Off-season } \\
\hline \multirow[t]{2}{*}{ Treatment } & Depth & $\mathrm{pH}$ & \multirow[t]{2}{*}{ DOC } & \multirow[t]{2}{*}{$\mathrm{N}-\mathrm{NO}_{3}^{-}$} & \multirow{2}{*}{$\mathrm{Cl}^{-}$} & \multirow{2}{*}{${\mathrm{S}-\mathrm{SO}_{4}}^{2-}$} & \multirow[t]{2}{*}{$\mathrm{P}$} & \multirow[t]{2}{*}{$\mathrm{F}^{-}$} \\
\hline & $\mathrm{cm}$ & & & & & & & \\
\hline Control & & $5.2 \pm(0.0)$ & $5.7 \pm(1.2)$ & $0.64 \pm(0.24)$ & $0.09 \pm(0.02)$ & $0.105 \pm(0.029)$ & $0.014 \pm(0.003)$ & $0.010 \pm(0.001)$ \\
\hline Silicate & $0-5$ & $5.8 \pm(0.1)$ & $4.5 \pm(1.1)$ & $0.44 \pm(0.06)$ & $0.07 \pm(0.01)$ & $0.134 \pm(0.090)$ & $0.008 \pm(0.001)$ & $0.026 \pm(0.009)$ \\
\hline Lime & & $6.3 \pm(0.0)$ & $6.5 \pm(2.1)$ & $0.57 \pm(0.29)$ & $0.07 \pm(0.01)$ & $0.084 \pm(0.021)$ & $0.016 \pm(0.004)$ & $0.020 \pm(0.005)$ \\
\hline Control & & $5.0 \pm(0.0)$ & $3.7 \pm(1.4)$ & $0.33 \pm(0.06)$ & $0.10 \pm(0.02)$ & $0.081 \pm(0.018)$ & $0.011 \pm(0.006)$ & $0.025 \pm(0.024)$ \\
\hline Silicate & $5-10$ & $5.3 \pm(0.1)$ & $4.0 \pm(1.7)$ & $0.37 \pm(0.13)$ & $0.06 \pm(0.02)$ & $0.131 \pm(0.024)$ & $0.015 \pm(0.008)$ & $0.010 \pm(0.002)$ \\
\hline Lime & & $5.5 \pm(0.1)$ & $5.4 \pm(1.0)$ & $0.73 \pm(0.69)$ & $0.06 \pm(0.01)$ & $0.113 \pm(0.020)$ & $0.016 \pm(0.005)$ & $0.011 \pm(0.003)$ \\
\hline Control & & $4.9 \pm(0.1)$ & $1.6 \pm(0.5)$ & $0.32 \pm(0.07)$ & $0.14 \pm(0.04)$ & $0.075 \pm(0.015)$ & $\mathrm{nd}^{*}$ & $0.010 \pm(0.000)$ \\
\hline Silicate & $10-20$ & $5.2 \pm(0.1)$ & $2.2 \pm(0.6)$ & $0.33 \pm(0.17)$ & $0.07 \pm(0.02)$ & $0.112 \pm(0.038)$ & $0.007 \pm(0.001)$ & $0.011 \pm(0.001)$ \\
\hline Lime & & $5.0 \pm(0.0)$ & $3.6 \pm(0.6)$ & $0.39 \pm(0.09)$ & $0.07 \pm(0.01)$ & $0.086 \pm(0.015)$ & $0.009 \pm(0.003)$ & $0.010 \pm(0.001)$ \\
\hline Control & & $5.1 \pm(0.2)$ & $5.0 \pm(0.0)$ & $0.22 \pm(0.09)$ & $0.08 \pm(0.03)$ & $0.154 \pm(0.011)$ & $0.007 \pm(0.001)$ & $\mathrm{nd}^{*}$ \\
\hline Silicate & $20-40$ & $4.7 \pm(0.3)$ & $4.6 \pm(0.6)$ & $0.24 \pm(0.06)$ & $0.08 \pm(0.02)$ & $0.119 \pm(0.004)$ & $0.009 \pm(0.003)$ & $\mathrm{nd}^{*}$ \\
\hline Lime & & $4.9 \pm(0.0)$ & $4.6 \pm(0.6)$ & $0.39 \pm(0.09)$ & $0.11 \pm(0.01)$ & $0.109 \pm(0.040)$ & $0.009 \pm(0.004)$ & $0.008 \pm(0.000)$ \\
\hline Control & & $5.0 \pm(0.0)$ & $3.9 \pm(0.5)$ & $0.23 \pm(0.06)$ & $0.09 \pm(0.02)$ & $0.128 \pm(0.023)$ & $0.014 \pm(0.000)$ & $\mathrm{nd}^{*}$ \\
\hline Silicate & $40-60$ & $4.9 \pm(0.0)$ & $3.7 \pm(0.9)$ & $0.30 \pm(0.13)$ & $0.10 \pm(0.02)$ & $0.137 \pm(0.023)$ & $0.004 \pm(0.001)$ & $\mathrm{nd}^{*}$ \\
\hline Lime & & $5.1 \pm(0.0)$ & $3.5 \pm(0.2)$ & $0.26 \pm(0.10)$ & $0.08 \pm(0.01)$ & $0.130 \pm(0.047)$ & $0.005 \pm(0.001)$ & $0.013 \pm(0.004)$ \\
\hline \multicolumn{2}{|c|}{ Treatments Depth } & $\mathrm{Al}$ & $\mathrm{Ca}$ & $\mathrm{Mg}$ & $\mathrm{K}$ & $\mathrm{Si}$ & $\mathrm{Fe}$ & 1 \\
\hline & $\mathrm{cm}$ & & & & & & & \\
\hline Control & & $0.39 \pm(0.35)$ & $0.18 \pm(0.04)$ & $0.13 \pm(0.03)$ & $0.36 \pm(0.13)$ & $0.35 \pm(0.30)$ & $0.049 \pm(0.041)$ & $1.83 \pm(0.69)$ \\
\hline Silicate & $0-5$ & $0.04 \pm(0.02)$ & $0.34 \pm(0.10)$ & $0.20 \pm(0.05)$ & $0.22 \pm(0.07)$ & $0.05 \pm(0.03)$ & $0.007 \pm(0.005)$ & $1.62 \pm(0.31)$ \\
\hline Lime & & $0.13 \pm(0.12)$ & $0.40 \pm(0.09)$ & $0.26 \pm(0.07)$ & $0.32 \pm(0.20)$ & $0.12 \pm(0.10)$ & $0.016 \pm(0.011)$ & $1.79 \pm(0.64)$ \\
\hline Control & & $0.08 \pm(0.06)$ & $0.15 \pm(0.05)$ & $0.09 \pm(0.02)$ & $0.13 \pm(0.05)$ & $0.08 \pm(0.05)$ & $0.011 \pm(0.008)$ & $0.95 \pm(0.19)$ \\
\hline Silicate & $5-10$ & $0.06 \pm(0.04)$ & $0.22 \pm(0.08)$ & $0.15 \pm(0.04)$ & $0.13 \pm(0.02)$ & $0.07 \pm(0$. & $0.009 \pm(0.009)$ & $1.26 \pm(0.24)$ \\
\hline Lime & & $0.13 \pm(0.06)$ & $0.22 \pm(0.05)$ & $0.16 \pm(0.05)$ & $0.15 \pm(0.09)$ & $0.11 \pm(0.05)$ & $0.020 \pm(0.011)$ & $1.44 \pm(0.43)$ \\
\hline Control & & $0.01 \pm(0.01)$ & $0.15 \pm(0.04)$ & $0.08 \pm(0.02)$ & $0.07 \pm(0.02)$ & $0.02 \pm(0.01)$ & $0.001 \pm(0.000)$ & $0.87 \pm(0.08)$ \\
\hline Silicate & $10-20$ & $0.02 \pm(0.01)$ & $0.16 \pm 10$ & $0.11 \pm 0$ & $0.10 \pm(0.06)$ & & 0.0 & $1.00 \pm$ \\
\hline Lime & & $0.03 \pm(0.02)$ & $0.17 \pm(0.04)$ & $0.12 \pm(0.02)$ & $0.09 \pm(0.05)$ & $0.06 \pm(0.02)$ & $0.003 \pm(0.002)$ & $1.00 \pm(0.19)$ \\
\hline Control & & $\mathrm{nd}^{*}$ & $0.14 \pm(0.03)$ & $0.09 \pm(0.02)$ & $0.06 \pm(0.03)$ & $0.02 \pm(0.00)$ & $0.000 \pm(0.000)$ & $0.92 \pm(0.00)$ \\
\hline Silicate & $20-40$ & $0.01 \pm(0.00)$ & $0.14 \pm(0.04)$ & $0.10 \pm(0.03)$ & $0.07 \pm(0.03)$ & $0.04 \pm(0.02)$ & $0.001 \pm(0.001)$ & $0.96 \pm(0.14)$ \\
\hline Lime & & $0.02 \pm(0.02)$ & $0.17 \pm(0.04)$ & $0.10 \pm(0.02)$ & $0.07 \pm(0.03)$ & $0.05 \pm(0.02)$ & $0.003 \pm(0.003)$ & $1.01 \pm(0.14)$ \\
\hline Control & & $0.00 \pm(0.00)$ & $0.12 \pm(0.04)$ & $0.07 \pm(0.02)$ & $0.04 \pm(0.01)$ & $0.03 \pm(0.02)$ & $0.000 \pm(0.000)$ & $0.63 \pm(0.23)$ \\
\hline Silicate & $40-60$ & $0.01 \pm(0.00)$ & $0.18 \pm(0.10)$ & $0.10 \pm(0.03)$ & $0.05 \pm(0.02)$ & $0.04 \pm(0.03)$ & $0.000 \pm(0.000)$ & $1.01 \pm(0.29)$ \\
\hline Lime & & $0.05 \pm(0.04)$ & $0.17 \pm(0.06)$ & $0.09 \pm(0.02)$ & $0.05 \pm(0.02)$ & $0.05 \pm(0.02)$ & $0.006 \pm(0.005)$ & $0.94 \pm(0.23)$ \\
\hline Treatments & Depth & $\mathrm{Mn}$ & $\mathrm{Cu}$ & $\mathrm{Zn}$ & $\mathrm{Na}$ & $\mathrm{N}^{-\mathrm{NO}_{2}^{-}}$ & $\mathrm{P}_{-}-\mathrm{PO}_{4}^{3-}$ & $\mathrm{S}$ \\
\hline & $\mathrm{cm}$ & & & & $10 \mathrm{~L}^{-1}$ & & & \\
\hline Control & & $0.007 \pm(0.002)$ & $0.0003 \pm(0.0001)$ & $0.0002 \pm(0.0000)$ & $0.04 \pm(0.03)$ & $0.006 \pm(0.004)$ & $0.0045 \pm(0.0001)$ & $0.123 \pm(0.032)$ \\
\hline Silicate & $0-5$ & $0.010 \pm(0.011)$ & $0.0002 \pm(0.0001)$ & $0.0002 \pm(0.0001)$ & $0.03 \pm(0.03)$ & $0.022 \pm(0.018)$ & $0.0027 \pm$ & $0.165 \pm(0.100)$ \\
\hline Lime & & $0.002 \pm(0.001)$ & $0.0003 \pm(0.0001)$ & $0.0000 \pm(0.0000)$ & $0.04 \pm(0.03)$ & $0.047 \pm(0.028)$ & $0.0052 \pm(0.0001)$ & $0.117 \pm(0.042)$ \\
\hline Control & & $0.005 \pm(0.001)$ & $0.0001 \pm(0.0000)$ & $0.0002 \pm(0.0001)$ & $0.03 \pm(0.03)$ & $0.003 \pm(0.000)$ & $0.0035 \pm(0.0018)$ & $0.086 \pm(0.020)$ \\
\hline Silicate & $5-10$ & $0.004 \pm(0.003)$ & $0.0001 \pm(0.0001)$ & $0.0002 \pm(0.0001)$ & $0.03 \pm(0.03)$ & $0.005 \pm(0.001)$ & $0.0048 \pm(0.0027)$ & $0.158 \pm(0.039)$ \\
\hline Lime & & $0.005 \pm(0.003)$ & $0.0002 \pm(0.0000)$ & $0.0001 \pm(0.0000)$ & $0.03 \pm(0.03)$ & $0.007 \pm(0.004)$ & $0.0053 \pm(0.0017)$ & $0.127 \pm(0.028)$ \\
\hline Control & & $0.006 \pm(0.003)$ & $0.0000 \pm(0.0000)$ & $0.0002 \pm(0.0000)$ & $0.03 \pm(0.03)$ & $0.004 \pm(0.000)$ & $\mathrm{nd}^{*}$ & $0.075 \pm(0.011)$ \\
\hline Silicate & $10-20$ & $0.003 \pm(0.002)$ & $0.0001 \pm(0.0000)$ & $0.0002 \pm(0.0001)$ & $0.03 \pm(0.03)$ & $0.005 \pm(0.000)$ & $0.0022 \pm(0.0002)$ & $0.126 \pm(0.031)$ \\
\hline Lime & & $0.012 \pm(0.006)$ & $0.0001 \pm(0.0000)$ & $0.0003 \pm(0.0002)$ & $0.03 \pm(0.03)$ & $0.003 \pm(0.000)$ & $0.0030 \pm(0.0010)$ & $0.096 \pm(0.009)$ \\
\hline Control & & $0.009 \pm(0.002)$ & $\mathrm{nd}^{*}$ & $0.0002 \pm(0.0001)$ & $0.05 \pm(0.00)$ & $0.006 \pm(0.000)$ & $0.0024 \pm(0.0003)$ & $0.162 \pm(0.049)$ \\
\hline Silicate & $20-40$ & $0.010 \pm(0.004)$ & $0.0000 \pm(0.0000)$ & $0.0002 \pm(0.0001)$ & $0.06 \pm(0.01)$ & $0.002 \pm(0.000)$ & $0.0028 \pm(0.0011)$ & $0.149 \pm(0.012)$ \\
\hline Lime & & $0.016 \pm(0.006)$ & $0.0001 \pm(0.0000)$ & $0.0002 \pm(0.0001)$ & $0.06 \pm(0.03)$ & $0.005 \pm(0.004)$ & $0.0029 \pm(0.0013)$ & $0.112 \pm(0.026)$ \\
\hline Control & & $0.010 \pm(0.006)$ & $0.0000 \pm(0.0000)$ & $0.0001 \pm(0.0000)$ & $0.05 \pm(0.01)$ & $0.003 \pm(0.001)$ & $0.0046 \pm(0.0000)$ & $0.123 \pm(0.013)$ \\
\hline Silicate & $40-60$ & $0.008 \pm(0.004)$ & $0.0000 \pm(0.0000)$ & $0.0001 \pm(0.0001)$ & $0.07 \pm(0.02)$ & $0.002 \pm(0.000)$ & $0.0014 \pm(0.0002)$ & $0.145 \pm(0.025)$ \\
\hline Lime & & $0.004 \pm(0.003)$ & $0.0001 \pm(0.0000)$ & $0.0001 \pm(0.0001)$ & $0.07 \pm(0.05)$ & $0.009 \pm(0.007)$ & $0.0016 \pm(0.0002)$ & $0.105 \pm(0.047)$ \\
\hline
\end{tabular}


ions) combine strongly with $\mathrm{Al}^{3+}$ (Merino et al., 1998), greatly reducing $a \mathrm{Al}^{3+}$ in solution. This is due to the high stability constant $(\log \beta)$ which exists between the complexes formed $\left(\log \beta \mathrm{AlF}_{2}{ }^{+}, \mathrm{AlF}_{2}{ }^{+}, \mathrm{AlF}_{3}, \mathrm{AlF}_{4}{ }^{-}, \mathrm{AlF}_{5}{ }^{2-}\right.$ and
$\mathrm{AlF}_{6}{ }^{3-}=6.13 ; 11.15 ; 15.00 ; 17.75 ; 19.37$ and 19.84 at 20 ${ }^{\circ} \mathrm{C}$, respectively). The higher the stability constant of an ionic pair, the lower the activity of the free forms of the ions in solution.

Table 5 - Acidity correctives, pH values, dissolved organic carbon (DOC), inorganic anions, cations and ionic strength (I), micronutrients, sodium, nitrite, phosphate and sulfur on soil solution at five depths of an Oxisol under no-tillage, in Season - Green-crop system. QL = quantification limit, mmol $\mathrm{L}^{-1}$.

\begin{tabular}{|c|c|c|c|c|c|c|c|c|}
\hline \multicolumn{9}{|c|}{ Season - Green-crop } \\
\hline \multirow{2}{*}{\multicolumn{2}{|c|}{$\begin{array}{c}\text { Treatments Depth } \\
\mathrm{cm}\end{array}$}} & \multirow[t]{2}{*}{$\mathrm{pH}$} & \multirow{2}{*}{\multicolumn{2}{|c|}{$\mathrm{N}^{-\mathrm{NO}_{3}}{ }^{-}$}} & \multirow{2}{*}{$\mathrm{Cl}^{-}$} & \multirow{2}{*}{$\mathrm{S}^{-\mathrm{SO}_{4}}{ }^{2-}$} & \multirow[t]{2}{*}{$P$} & \multirow[t]{2}{*}{$\mathrm{F}^{-}$} \\
\hline & & & & & & & & \\
\hline Control & \multirow{3}{*}{$0-5$} & $5.3 \pm(0.0)$ & $4.8 \pm(1.6)$ & $0.73 \pm(0.70)$ & $0.08 \pm(0.03)$ & $0.150 \pm(0.047)$ & $0.010 \pm(0.003)$ & $0.009 \pm(0.001)$ \\
\hline Silicate & & $6.1 \pm(0.0)$ & $5.2 \pm(1.3)$ & $0.34 \pm(0.13)$ & $0.08 \pm(0.02)$ & $0.120 \pm(0.048)$ & $0.014 \pm(0.003)$ & $0.024 \pm(0.012)$ \\
\hline Lime & & $5.9 \pm(0.0)$ & $7.7 \pm(1.1)$ & $0.61 \pm(0.23)$ & $0.07 \pm(0.02)$ & $0.140 \pm(0.036)$ & $0.018 \pm(0.003)$ & $0.013 \pm(0.004)$ \\
\hline Control & \multirow{3}{*}{$5-10$} & $5.1 \pm(0.1)$ & $3.7 \pm(0.6)$ & $0.34 \pm(0.15)$ & $0.11 \pm(0.03)$ & $0.117 \pm(0.022)$ & $0.013 \pm(0.006)$ & $0.009 \pm(0.001)$ \\
\hline Silicate & & $5.5 \pm(0.1)$ & $4.8 \pm(0.5)$ & $0.29 \pm(0.17)$ & $0.09 \pm(0.04)$ & $0.127 \pm(0.028)$ & $0.011 \pm(0.001)$ & $0.014 \pm(0.003)$ \\
\hline Lime & & $5.0 \pm(0.0)$ & $5.0 \pm(0.7)$ & $0.35 \pm(0.17)$ & $0.07 \pm(0.03)$ & $0.142 \pm(0.033)$ & $0.013 \pm(0.001)$ & $0.008 \pm(0.000)$ \\
\hline Control & \multirow{3}{*}{$10-20$} & $4.8 \pm(0.1)$ & $2.5 \pm(0.8)$ & $0.30 \pm(0.13)$ & $0.10 \pm(0.03)$ & $0.094 \pm(0.037)$ & $0.006 \pm(0.001)$ & $0.008 \pm(0.000)$ \\
\hline Silicate & & $5.0 \pm(0.0)$ & $2.5 \pm(0.4)$ & $0.22 \pm(0.07)$ & $0.08 \pm(0.02)$ & $0.132 \pm(0.023)$ & $0.008 \pm(0.000)$ & $0.008 \pm(0.000)$ \\
\hline Lime & & $4.8 \pm(0.0)$ & $2.6 \pm(0.4)$ & $0.33 \pm(0.12)$ & $0.13 \pm(0.11)$ & $0.351 \pm(0.363)$ & $0.007 \pm(0.002)$ & $0.009 \pm(0.000)$ \\
\hline Control & \multirow{3}{*}{$20-40$} & $5.0 \pm(0.0)$ & $4.7 \pm(0.3)$ & $0.60 \pm(0.49)$ & $0.10 \pm(0.01)$ & $0.122 \pm(0.017)$ & $0.011 \pm(0.004)$ & $0.022 \pm(0.000)$ \\
\hline Silicate & & $5.1 \pm(0.1)$ & $4.7 \pm(0.1)$ & $0.27 \pm(0.07)$ & $0.11 \pm(0.05)$ & $0.169 \pm(0.040)$ & $0.009 \pm(0.003)$ & $\mathrm{nd}^{*}$ \\
\hline Lime & & $4.8 \pm(0.0)$ & $5.2 \pm(0.4)$ & $0.32 \pm(0.16)$ & $0.10 \pm(0.04)$ & $0.135 \pm(0.020)$ & $0.007 \pm(0.002)$ & $\mathrm{nd}^{*}$ \\
\hline Control & \multirow{3}{*}{$40-60$} & $5.2 \pm(0.1)$ & $4.0 \pm(0.7)$ & $0.19 \pm(0.03)$ & $0.08 \pm(0.02)$ & $0.134 \pm(0.029)$ & $0.008 \pm 10.0$ & $\mathrm{nd}^{*}$ \\
\hline Silicate & & $5.0 \pm(0.0)$ & $3.5 \pm(0.7)$ & $0.22 \pm(0.07)$ & $0.07 \pm(0.02)$ & $0.165 \pm(0.035)$ & $0.004 \pm(0.001)$ & $0.012 \pm(0.000)$ \\
\hline Lime & & $5.0 \pm(0.0)$ & $3.8 \pm(0.3)$ & $0.21 \pm(0.05)$ & $0.08 \pm(0.01)$ & $0.141 \pm(0.029)$ & $0.005 \pm(0.000)$ & $\mathrm{nd}^{*}$ \\
\hline \multirow[t]{2}{*}{ Treatments } & Depth & $\mathrm{Al}$ & $\mathrm{Ca}$ & $\mathrm{Mg}$ & $\mathrm{K}$ & $\mathrm{Si}$ & $\mathrm{Fe}$ & 1 \\
\hline & \multirow[t]{2}{*}{$\mathrm{cm}$} & & & & & & & \\
\hline Control & & $0.23 \pm(0.13)$ & $0.21 \pm(0.07)$ & $0.16 \pm(0.07)$ & $0.31 \pm(0.13)$ & $0.20 \pm(0.12)$ & $0.024 \pm(0.013)$ & $1.26 \pm(0.37)$ \\
\hline Silicate & \multirow[t]{2}{*}{$0-5$} & $0.04 \pm(0.01)$ & $0.37 \pm(0.18)$ & $0.20 \pm(0.08)$ & $0.25 \pm(0.16)$ & $0.08 \pm(0.04)$ & $0.006 \pm 10$. & $1.57 \pm(0.58)$ \\
\hline Lime & & $0.36 \pm(0.20)$ & $0.26 \pm(0.08)$ & $0.22 \pm(0.04)$ & $0.42 \pm(0.17)$ & $0.32 \pm(0.20)$ & $0.044 \pm 10.0$ & $1.92 \pm(0.65)$ \\
\hline Control & \multirow{3}{*}{$5-10$} & $0.10 \pm(0.08)$ & $0.17 \pm(0.05)$ & $0.12 \pm(0.03)$ & $0.11 \pm(0.03)$ & $0.09 \pm(0.05)$ & $0.011 \pm 10$. & $1.14 \pm(0.24)$ \\
\hline Silicate & & $0.10 \pm(0.09)$ & $0.22 \pm(0.08)$ & $0.16 \pm(0.04)$ & $0.10 \pm(0.02)$ & $0.09 \pm(0.09)$ & $0.014 \pm(0.015)$ & $1.25 \pm(0.32)$ \\
\hline Lime & & $0.09 \pm(0.07)$ & $0.16 \pm(0.06)$ & $0.11 \pm(0.03)$ & $0.18 \pm(0.07)$ & $0.08 \pm(0.05)$ & $0.012 \pm(0.011)$ & $1.15 \pm(0.31)$ \\
\hline Control & \multirow{3}{*}{$10-20$} & $0.03 \pm(0.03)$ & $0.16 \pm(0.05)$ & $0.09 \pm(0.03)$ & $0.07 \pm(0.02)$ & $0.04 \pm(0.03)$ & $0.004 \pm(0.005)$ & $0.92 \pm(0.22)$ \\
\hline Silicate & & $0.01 \pm(0.01)$ & $0.14 \pm($ & $0.10 \pm($ & $0.06 \pm 1 C$ & $0.03 \pm 1 C$ & 1) & $0.91 \pm$ \\
\hline Lime & & $0.02 \pm(0.02)$ & $0.12 \pm(0.04)$ & $0.08 \pm(0.01)$ & $0.09 \pm(0.03)$ & $0.04 \pm(0.02)$ & $0.003 \pm 10$. & $0.90 \pm(0.15)$ \\
\hline Control & \multirow{3}{*}{$20-40$} & $0.01 \pm(0.01)$ & $0.20 \pm(0.08)$ & $0.11 \pm(0.04)$ & $0.06 \pm(0.02)$ & $0.03 \pm(0.01)$ & $0.001 \pm(0.001)$ & $1.20 \pm(0.48)$ \\
\hline Silicate & & $0.02 \pm(0.00)$ & $0.21 \pm(0.13)$ & $0.12 \pm(0.06)$ & $0.06 \pm(0.02)$ & $0.04 \pm(0.03)$ & $0.001 \pm(0.001)$ & $1.20 \pm(0.53)$ \\
\hline Lime & & $0.01 \pm(0.00)$ & $0.13 \pm(0.06)$ & $0.07 \pm(0.02)$ & $0.07 \pm(0.02)$ & $0.03 \pm(0.02)$ & $0.000 \pm(0.000)$ & $0.82 \pm(0.16)$ \\
\hline Control & & $0.02 \pm(0.01)$ & $0.19 \pm(0.08)$ & $0.10 \pm(0.04)$ & $0.05 \pm(0.02)$ & $0.04 \pm(0.01)$ & $0.002 \pm(0.001)$ & $0.98 \pm(0.27)$ \\
\hline Silicate & $40-60$ & $0.02 \pm(0.00)$ & $0.17 \pm(0.07)$ & $0.10 \pm(0.04)$ & $0.03 \pm(0.01)$ & $0.03 \pm(0.02)$ & $0.003 \pm(0.003)$ & $0.97 \pm(0.27)$ \\
\hline Lime & & $0.01 \pm(0.00)$ & $0.13 \pm(0.04)$ & $0.07 \pm(0.02)$ & $0.05 \pm(0.02)$ & $0.03 \pm(0.02)$ & $0.001 \pm(0.001)$ & $0.83 \pm(0.24)$ \\
\hline Treatments & Depth & $\mathrm{Mn}$ & $\mathrm{Cu}$ & $\mathrm{Zn}$ & $\mathrm{Na}$ & $\mathrm{N}-\mathrm{NO}_{2}^{-}$ & $\mathrm{P}_{-}-\mathrm{PO}_{4}^{3-}$ & $S$ \\
\hline & $\mathrm{cm}$ & & & & $\mathrm{OI}$ & & & \\
\hline Control & & $0.006 \pm(0.003)$ & & $0.0005 \pm(0.0003)$ & $0.05 \pm(0.01)$ & $0.017 \pm(0.007)$ & $0.0032 \pm(0.0009)$ & $0.150 \pm(0.03)$ \\
\hline Silicate & $0-5$ & $0.002 \pm(0.002)$ & $0.0003 \pm 10$ & $0.0001 \pm(0.0000)$ & $0.03 \pm(0.03)$ & $0.022 \pm(0.016)$ & $0.0046 \pm 10$ & $0.158 \pm(0.072)$ \\
\hline Lime & & $0.003 \pm(0.001)$ & $0.0004 \pm(0.0001)$ & $0.0001 \pm(0.0000)$ & $0.03 \pm(0.03)$ & $0.038 \pm(0.027)$ & $0.0059 \pm(0.0011)$ & $0.152 \pm(0.037)$ \\
\hline Control & & $0.006 \pm(0.003)$ & $0.0001 \pm(0.0000)$ & $0.0003 \pm(0.0002)$ & $0.04 \pm(0.03)$ & $0.005 \pm(0.002)$ & $0.0041 \pm(0.0020)$ & $0.129 \pm(0.025)$ \\
\hline Silicate & $5-10$ & $0.007 \pm(0.007)$ & $0.0002 \pm(0.0001)$ & $0.0003 \pm(0.0002)$ & $0.04 \pm(0.02)$ & $0.011 \pm(0.006)$ & $0.0035 \pm(0.0004)$ & $0.153 \pm(0.040)$ \\
\hline Lime & & $0.008 \pm(0.002)$ & $0.0001 \pm(0.0000)$ & $0.0003 \pm(0.0000)$ & $0.03 \pm(0.03)$ & $0.002 \pm(0.000)$ & $0.0043 \pm(0.0002)$ & $0.161 \pm(0.026)$ \\
\hline Control & & $0.009 \pm(0.002)$ & $0.0000 \pm(0.0000)$ & $0.0002 \pm(0.0001)$ & $0.03 \pm(0.03)$ & $0.001 \pm(0.000)$ & $0.0020 \pm(0.0002)$ & $0.099 \pm(0.036)$ \\
\hline Silicate & $10-20$ & $0.007 \pm(0.001)$ & $0.0001 \pm(0.0000)$ & $0.0003 \pm(0.0001)$ & $0.03 \pm(0.03)$ & $0.002 \pm(0.000)$ & $0.0027 \pm(0.0000)$ & $0.142 \pm(0.024)$ \\
\hline Lime & & $0.016 \pm(0.004)$ & $0.0001 \pm(0.0000)$ & $0.0017 \pm(0.0018)$ & $0.03 \pm(0.02)$ & $\mathrm{nd}^{*}$ & $0.0022 \pm(0.0006)$ & $0.108 \pm(0.013)$ \\
\hline Control & & $0.016 \pm(0.007)$ & $0.0000 \pm(0.0000)$ & $0.0002 \pm(0.0001)$ & $0.06 \pm(0.03)$ & $0.003 \pm(0.001)$ & $0.0036 \pm(0.0011)$ & $0.140 \pm(0.036)$ \\
\hline Silicate & $20-40$ & $0.015 \pm(0.003)$ & $0.0000 \pm(0.0000)$ & $0.0001 \pm(0.0001)$ & $0.06 \pm(0.02)$ & $0.004 \pm(0.001)$ & $0.0029 \pm(0.0010)$ & $0.180 \pm(0.066)$ \\
\hline Lime & & $0.014 \pm(0.003)$ & $0.0001 \pm(0.0000)$ & $0.0001 \pm(0.0001)$ & $0.06 \pm(0.02)$ & $0.002 \pm(0.000)$ & $0.0022 \pm(0.0005)$ & $0.113 \pm(0.019)$ \\
\hline Control & & $0.007 \pm(0.005)$ & $0.0000 \pm(0.0000)$ & $0.0001 \pm(0.0001)$ & $0.06 \pm(0.03)$ & $0.002 \pm(0.001)$ & $0.0027 \pm(0.0009)$ & $0.158 \pm(0.070)$ \\
\hline Silicate & $40-60$ & $0.005 \pm(0.002)$ & $0.0001 \pm(0.0000)$ & $0.0001 \pm(0.0000)$ & $0.06 \pm(0.02)$ & $0.005 \pm(0.000)$ & $0.0013 \pm(0.0002)$ & $0.163 \pm(0.037)$ \\
\hline Lime & & $0.004 \pm(0.001)$ & $0.0000 \pm(0.0000)$ & $0.0001 \pm(0.0001)$ & $0.06 \pm(0.02)$ & $0.002 \pm(0.000)$ & $0.0017 \pm(0.0000)$ & $0.132 \pm(0.051)$ \\
\hline
\end{tabular}


Bessho and Bell (1992) found that the addition of lime $\left(\mathrm{CaCO}_{3}\right)$ and the leaves of leguminous plants and straw bean plants in samples of the surface layer of an Oxisol reduced the activity of aluminum monomeric species, which corresponded to $20 \%$ of the total $\mathrm{Al}$ concentration in solution. This occurrence is attributable to the formation of aluminum ion complexes with organic compounds released by plant residues, as well as $\mathrm{Al}^{3+}$

Table 6 - Acidity correctives, pH values, dissolved organic carbon (DOC), inorganic anions, cations and ionic strength (I), micronutrients, sodium, nitrite, phosphate and sulfur on soil solution at five depths of an Oxisol under no-tillage, in Season - Fallow system. QL $=$ quantification limit, $\mathrm{mmol}^{-1}$.

\begin{tabular}{|c|c|c|c|c|c|c|c|c|}
\hline \multicolumn{9}{|c|}{ Season - Fallow } \\
\hline \multirow[t]{2}{*}{ Treatments } & Depth & \multirow[t]{2}{*}{$\mathrm{pH}$} & \multirow[t]{2}{*}{ DOC } & \multirow[t]{2}{*}{$\mathrm{N}^{-\mathrm{NO}_{3}}{ }^{-}$} & $\mathrm{Cl}^{-}$ & \multirow[t]{2}{*}{ 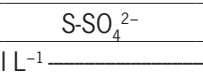 } & \multirow[t]{2}{*}{$P$} & \multirow[t]{2}{*}{$\mathrm{F}^{-}$} \\
\hline & $\mathrm{cm}$ & & & & $\mathrm{mmo}$ & & & \\
\hline Control & & $4.8 \pm(0.0)$ & $3.1 \pm(1.5)$ & $0.45 \pm(0.33)$ & $0.10 \pm(0.05)$ & $0.178 \pm(0.005)$ & $0.007 \pm(0.005)$ & $0.008 \pm(0.001)$ \\
\hline Silicate & $0-5$ & $6.4 \pm(0.0)$ & $5.5 \pm(0.5)$ & $0.97 \pm(0.69)$ & $0.09 \pm(0.01)$ & $0.212 \pm(0.005)$ & $0.020 \pm(0.005)$ & $0.030 \pm(0.014)$ \\
\hline Lime & & $6.1 \pm(0.1)$ & $5.8 \pm(0.8)$ & $0.61 \pm(0.24)$ & $0.07 \pm(0.02)$ & $0.185 \pm(0.005)$ & $0.021 \pm(0.005)$ & $0.015 \pm(0.003)$ \\
\hline Control & & $4.6 \pm(0.1)$ & $3.2 \pm(0.6)$ & $0.35 \pm(0.09)$ & $0.14 \pm(0.08)$ & $0.138 \pm(0.005)$ & $0.013 \pm(0.005)$ & $0.009 \pm(0.002)$ \\
\hline Silicate & $5-10$ & $5.5 \pm(0.1)$ & $4.5 \pm(1.3)$ & $0.38 \pm(0.10)$ & $0.16 \pm(0.13)$ & $0.226 \pm(0.005)$ & $0.014 \pm(0.005)$ & $0.017 \pm(0.004)$ \\
\hline Lime & & $5.3 \pm(0.1)$ & $3.5 \pm(0.5)$ & $0.39 \pm(0.02)$ & $0.09 \pm(0.03)$ & $0.197 \pm(0.005)$ & $0.014 \pm(0.005)$ & $0.009 \pm(0.001)$ \\
\hline Control & & $4.4 \pm(0.0)$ & $1.8 \pm(0$ & $0.44 \pm(0.19)$ & $0.19 \pm(0.06)$ & $0.085 \pm(0.005)$ & $0.007 \pm(0.005)$ & $0.009 \pm(0.001)$ \\
\hline Silicate & $10-20$ & $4.8 \pm 1 C$ & $1.6 \pm(C$ & $0.48 \pm 10$ & $0.17 \pm(0.06)$ & $0.152 \pm(0.005)$ & $0.003 \pm 10.0$ & $0.009 \pm(0.000)$ \\
\hline Lime & & $4.8 \pm(0.1)$ & $1.6 \pm(0.2)$ & $0.51 \pm(0.06)$ & $0.18 \pm(0.05)$ & $0.124 \pm(0.005)$ & $0.004 \pm(0.005)$ & $0.010 \pm(0.001)$ \\
\hline Control & & $4.6 \pm(0.1)$ & $4.9 \pm(0.3)$ & $0.38 \pm(0.07)$ & $0.16 \pm(0.03)$ & $0.106 \pm(0.005)$ & $0.011 \pm(0.005)$ & $0.009 \pm(0.000)$ \\
\hline Silicate & $20-40$ & $4.9 \pm(0.1)$ & $4.7 \pm 10$ & $0.46 \pm 10$. & $0.23 \pm(0.12)$ & $0.158 \pm 10$. & $0.012 \pm 10$. & $0.012 \pm(0.000)$ \\
\hline Lime & & $4.8 \pm(0.1)$ & $4.4 \pm(0.6)$ & $0.35 \pm(0.02)$ & $0.19 \pm(0.05)$ & $0.176 \pm(0.005)$ & $0.010 \pm(0.005)$ & $0.009 \pm(0.000)$ \\
\hline Control & & $4.6 \pm(0.3)$ & $3.6 \pm(0.6)$ & $0.22 \pm(0.06)$ & $0.09 \pm(0.02)$ & $0.141 \pm(0.005)$ & $0.009 \pm(0.005)$ & $0.010 \pm(0.000)$ \\
\hline Silicate & $40-60$ & $4.7 \pm(0.2)$ & $3.3 \pm(0.3)$ & $0.35 \pm(0.19)$ & $0.10 \pm(0.05)$ & $0.175 \pm(0.005)$ & $0.008 \pm 10$. & $0.010 \pm(0.000)$ \\
\hline Lime & & $4.6 \pm(0.4)$ & $3.1 \pm(0.0)$ & $0.24 \pm(0.05)$ & $0.10 \pm(0.02)$ & $0.173 \pm(0.005)$ & $0.012 \pm(0.005)$ & $\mathrm{nd}^{*}$ \\
\hline \multirow[t]{2}{*}{ Treatments } & Depth & $\mathrm{Al}$ & $\mathrm{Ca}$ & $\mathrm{Mg}$ & $\mathrm{K}$ & $\mathrm{Si}$ & $\mathrm{Fe}$ & 1 \\
\hline & $\mathrm{cm}$ & & & & & & & \\
\hline Control & & $0.07 \pm(0.05)$ & $0.16 \pm(0.07)$ & $0.13 \pm(0.07)$ & & $0.09 \pm(0.05)$ & $0.012 \pm(0.012)$ & $1.58 \pm(0.56)$ \\
\hline Silicate & $0-5$ & $0.06 \pm 1$ & & & & & & \\
\hline Lime & & $0.14 \pm(0.04)$ & $0.45 \pm 10$ & $0.31 \pm 10$ & $0.51 \pm(0.23)$ & $0.13 \pm$ & 0.0 & $.01)$ \\
\hline Control & & $0.01 \pm(0.00)$ & $0.13 \pm(0.07)$ & $0.09 \pm(0.04)$ & $0.24 \pm(0.10)$ & $0.03 \pm(0.02)$ & $0.001 \pm(0.000)$ & $1.14 \pm(0.34)$ \\
\hline Silicate & $5-10$ & $0.04 \pm(0.04)$ & $0.31 \pm(0.07)$ & $0.19 \pm(0.06)$ & $0.16 \pm(0.06)$ & $0.04 \pm(0.03)$ & 0.0 & $.46)$ \\
\hline Lime & & $0.04 \pm 0$. & $0.24 \pm$ & $0.17 \pm 10$ & 0.1 & & 3) & \\
\hline Control & & $0.01 \pm(0.00)$ & $0.14 \pm(0.07)$ & $0.09 \pm 1 C$ & $4 \pm(0.04)$ & $0.02 \pm 10$ & 00) & .27) \\
\hline Silicate & $10-20$ & $0.01 \pm(0.01)$ & $0.22 \pm(0.07)$ & $0.14 \pm(0.04)$ & $0.09 \pm(0.03)$ & $0.02 \pm 10$ & 00) & $1.39 \pm($ \\
\hline Lime & & $0.02 \pm(0.03)$ & $0.20 \pm(0.07)$ & $0.14 \pm(0.04)$ & $0.09 \pm(0.01)$ & $0.02 \pm(0.01)$ & $2 \pm 10$ & $1.37 \pm(0.30)$ \\
\hline Control & & $0.00 \pm(0.00)$ & & $0.07 \pm(0.02)$ & $0.08 \pm(0.01)$ & $0.02 \pm 10$ & 00) & $1.00 \pm(0$ \\
\hline Silicate & $20-40$ & $0.01 \pm(0.01)$ & $0.24 \pm 10$ & $0.14 \pm($ & $0.07 \pm(0.01)$ & 0.03 & 0.0 & $1.46 \pm$ \\
\hline Lime & & $0.01 \pm(0.00)$ & $0.22 \pm(0.07)$ & $0.14 \pm(0.05)$ & $0.08 \pm(0.01)$ & $0.03 \pm 10$ & $0.000 \pm(0.000)$ & $1.40 \pm(0.21)$ \\
\hline Control & & $0.00 \pm(0.00)$ & $0.11 \pm(0.07)$ & $0.06 \pm(0.02)$ & $0.07 \pm(0.01)$ & $0.04 \pm(0.03)$ & $0.001 \pm(0.000)$ & $0.86 \pm(0.10)$ \\
\hline Silicate & $40-60$ & $0.01 \pm(0.00)$ & & $0.10 \pm(0.05)$ & $0.05 \pm(0.02)$ & $0.02 \pm(0.01)$ & $0.000 \pm(0.000)$ & $1.14 \pm(0.42)$ \\
\hline Lime & & $0.02 \pm(0.01)$ & $0.12 \pm(0.07)$ & $0.07 \pm(0.03)$ & $0.06 \pm(0.01)$ & $0.03 \pm(0.01)$ & $0.003 \pm(0.003)$ & $0.88 \pm(0.35)$ \\
\hline \multirow[t]{2}{*}{ Treatments } & Depth & $\mathrm{Mn}$ & $\mathrm{Cu}$ & $\mathrm{Zn}$ & $\mathrm{Na}$ & $\mathrm{N}-\mathrm{NO}_{2}^{-}$ & $\mathrm{P}_{-} \mathrm{PO}_{4}^{3-}$ & $S$ \\
\hline & $\mathrm{cm}$ & & & & & & & \\
\hline Control & & $0.010 \pm(0.004)$ & $0.0001 \pm(0.0000)$ & $0.0003 \pm(0.0000)$ & $5 \pm(0.03)$ & $0.020 \pm(0.018)$ & $0.0021 \pm(0.0005)$ & $0.212 \pm(0.077)$ \\
\hline Silicate & $0-5$ & $0.001 \pm(0.001)$ & $0.0003 \pm(0.0001)$ & $0.0000 \pm(0.0000)$ & $0.05 \pm(0.03)$ & $0.068 \pm(0.055)$ & $0.0064 \pm(0.0016)$ & $0.265 \pm(0.049)$ \\
\hline Lime & & $0.002 \pm(0.001)$ & $0.0004 \pm(0.0001)$ & $0.0000 \pm(0.0000)$ & $0.04 \pm(0.03)$ & $0.047 \pm(0.022)$ & $0.0067 \pm(0.0010)$ & $0.238 \pm(0.059)$ \\
\hline Control & & $0.018 \pm(0.003)$ & $0.0001 \pm(0.0000)$ & $0.0008 \pm(0.0001)$ & $0.04 \pm(0.03)$ & & $0.0041 \pm(0.0008)$ & $0.163 \pm(0.062)$ \\
\hline Silicate & $5-10$ & $0.003 \pm(0.002)$ & $0.0001 \pm(0.0000)$ & $0.0001 \pm(0.0001)$ & $0.03 \pm(0.02)$ & $0.004 \pm(0.001)$ & $0.0045 \pm(0.0024)$ & $0.285 \pm(0.107)$ \\
\hline Lime & & $0.005 \pm(0.003)$ & $0.0001 \pm(0.0000)$ & $0.0002 \pm(0.0001)$ & $0.04 \pm(0.03)$ & $0.007 \pm(0.000)$ & $0.0046 \pm(0.0010)$ & $0.257 \pm(0.041)$ \\
\hline Control & & $0.024 \pm(0.003)$ & $0.0001 \pm(0.0000)$ & $0.0009 \pm(0.0001)$ & $0.03 \pm(0.02)$ & $\mathrm{nd}^{*}$ & $0.0022 \pm(0.0006)$ & $0.096 \pm(0.019)$ \\
\hline Silicate & $10-20$ & $0.011 \pm(0.007)$ & $0.0000 \pm(0.0000)$ & $0.0007 \pm(0.0003)$ & $0.03 \pm(0.02)$ & $\mathrm{nd}^{*}$ & $0.0011 \pm(0.0000)$ & $0.162 \pm(0.018)$ \\
\hline Lime & & $0.013 \pm(0.006)$ & $0.0000 \pm(0.0000)$ & $0.0003 \pm(0.0001)$ & $0.03 \pm(0.03)$ & $\mathrm{nd}^{*}$ & $0.0014 \pm(0.0002)$ & $0.139 \pm(0.012)$ \\
\hline Control & & $0.030 \pm(0.003)$ & $0.0001 \pm(0.0000)$ & $0.0003 \pm(0.0001)$ & $0.06 \pm(0.01)$ & $0.003 \pm(0.000)$ & $0.0037 \pm(0.0010)$ & $0.125 \pm(0.043)$ \\
\hline Silicate & $20-40$ & $0.026 \pm(0.014)$ & $0.0000 \pm(0.0000)$ & $0.0002 \pm(0.0001)$ & $0.06 \pm(0.02)$ & $n d^{*}$ & $0.0040 \pm(0.0005)$ & $0.177 \pm(0.007)$ \\
\hline Lime & & $0.021 \pm(0.003)$ & $0.0000 \pm(0.0000)$ & $0.0002 \pm(0.0001)$ & $0.07 \pm(0.04)$ & $0.003 \pm(0.000)$ & $0.0033 \pm(0.0010)$ & $0.219 \pm(0.027)$ \\
\hline Control & & $0.008 \pm(0.001)$ & $0.0000 \pm(0.0000)$ & $0.0001 \pm(0.0001)$ & $0.06 \pm(0.02)$ & $0.003 \pm(0.000)$ & $0.0029 \pm(0.0005)$ & $0.161 \pm(0.025)$ \\
\hline Silicate & $40-60$ & $0.007 \pm(0.004)$ & $\mathrm{nd}^{*}$ & $0.0002 \pm(0.0001)$ & $0.06 \pm(0.03)$ & $0.002 \pm(0.000)$ & $0.0027 \pm(0.0008)$ & $0.194 \pm(0.033)$ \\
\hline Lime & & $0.010 \pm(0.005)$ & $0.0000 \pm(0.0000)$ & $0.0001 \pm(0.0000)$ & $0.04 \pm(0.01)$ & $\mathrm{nd}^{*}$ & $0.0040 \pm(0.0007)$ & $0.255 \pm(0.034)$ \\
\hline
\end{tabular}

Values between parentheses mean the average deviation $(n=4) ; \mathrm{nd}^{*}=$ not detected: $\mathrm{QL}: \mathrm{F}^{-}=0.0026 ; \mathrm{N}^{-} \mathrm{NO}_{2}{ }^{-}=0.0011$. 
combinations with hydroxyl ions $\left(\mathrm{OH}^{-}\right)$, residues of lime reaction in the soil surface, both of which contribute significantly to reducing $\mathrm{Al}^{3+}$ activity in solution.

Correlations between $\mathrm{Al} t$ and $a \mathrm{Al}^{3+}$ were positive in all layers with higher coefficients of determination in the first three layers (Figure 1). The low values of $a \mathrm{Al}^{3+}$ compared to the Alt are due to complexation of $\mathrm{Al}^{3+}$ by organic anions, which decreases this ion's activity in solution, especially in the topsoil layers (Vance et al., 1996). In the deeper layers, where there is little OM activity, low correlation may be related to differences in the concentrations of organic compounds and fluoride $\left(\mathrm{F}^{-}\right)$in solution. Failure to correct the $\mathrm{pH}$ in the subsurface, taking into account the fact that the application of correctives occurred in the top surface layers, may also explain the low correlations being related to differences between the concentrations of hydroxyls $\left(\mathrm{OH}^{-}\right)$and fluoride $\left(\mathrm{F}^{-}\right)$.

In accordance with the findings of Zambrosi et al. (2008), aluminum was detected in lower quantities in the free form $\left(\mathrm{Al}^{3+}\right)$, and in greater quantities when forming complexes in the soil solution (Figure 2) regardless of corrective or crop system adopted. According to Drabeck et al. (2005), the toxicity threat to the plant decreases in the following order: $\mathrm{Al}_{13}$ polymers (except for phosphates and silicates), $\mathrm{Al}^{3+}, \mathrm{Al}(\mathrm{OH})^{2+}, \mathrm{Al}(\mathrm{OH})_{2}{ }^{+}$, $\mathrm{Al}(\mathrm{OH})_{3}, \mathrm{Al}(\mathrm{OH})_{4}{ }^{-}$and $\mathrm{AlSO}_{4}{ }^{+}$. However, the toxicity of the last four species is not always considered. The free form which is toxic to plants $\left(\mathrm{Al}^{+3}\right)$ was not very representative, even in those soil samples which received neither lime nor silicate (Figure 2). The highest value was $15 \%$, observed in samples under the Season - Off-season system - at a depth of $20-40 \mathrm{~cm}$ in the control treatment (Figure 2), probably due to the non-formation of ionic pairing with $\mathrm{F}^{-}$(Table 3). Overall, this species was found with more frequency in the deepest two layers, (the major part in the 40-60 cm layer) where the performance of $\mathrm{OM}$ and correctives is generally poor.

According to an average based on all the treatments, the Al-DOC species was predominant in the majority of cases, surpassed by the Al-F species only in the 0-20 cm layer under two systems - Season - Forage and Season - Fallow (Figure 2), and the Al-OH species in the upper surface layer $(0-5 \mathrm{~cm})$ of the Season - Fallow system under which both correctives were applied; the Season - Green-crop system, under which calcium-magnesium silicates were added; and Season - Off-season system under which only lime was added (Figure 2).

The Season - Fallow system does not maintain vegetation in the soil during the off-season, which reduces the supply of both OM in the soil and, consequently, organic acids mineralized during $\mathrm{OM}$ decomposition. The organic anions derived from these acids form complexes with $\mathrm{Al}$ (Al-DOC), and this accounts for the higher percentage of Al-OH species, compared to the Al-DOC species, in the upper layers. OM decomposition under the Season - Fallow system may have occurred gradually, and is possibly explained by several factors, including higher Si in the grasses, which adds rigidity to the plant structure and retards its rate of decomposition (Ramos et al., 2006). In the deeper layers of the soil, where there is no strong presence of organic compounds or correctives, the $\mathrm{F}^{-}$will prevail when combining with $\mathrm{Al}$ (Zambrosi et al., 2007), which also depends on the concentration and activity of the fluoride in solution.

Although, in general, the greater part of the root system of crops of agricultural interest is to be found in the first $10 \mathrm{~cm}$ below the surface, the root system can extend down to $40 \mathrm{~cm}$. The complexation of Al by DOC, up to a depth of $40 \mathrm{~cm}$, remained unaffected by acidity correctives treatments (Zambrosi et al., 2008). Alleoni et al. (2010) observed that the proportion of Al-DOC in the soil solution under NT was approximately $85 \%$ in the layer from 0.0 to $0.2 \mathrm{~m}$, and this proportion was only slightly affected by the application of lime on the surface. Adams et al. (1999) found that the amount of Al-DOC positively correlated with concentrations of dissolved humic substances (high molecular weight) in acidic soils in New Zealand. Thus, the dissolved organic anions gain stability from complexing with $\mathrm{Al}$ in solution, and its contribution depends on the OM activity in the soil.

Generally, all species bind to the $\mathrm{F}^{-}$ion and organic compounds (DOC), and precipitate as $\mathrm{Al}(\mathrm{OH})_{3}$. Although $\mathrm{Al}_{13}\left(\mathrm{Al}^{3+}\right)$ is the most toxic, or most damaging, species to plant root systems it is not usually present in high numbers in soils (Drabeck et al., 2005), as was revealed by our study. Even under the natural conditions of acidic soils where, in solution, low to moderate concentrations of $\mathrm{Al}^{3+}$ of the complexes formed can be observed between aluminum and fluoride $(\mathrm{Al}-\mathrm{F})$ and aluminum and dissolved organic carbon (Al-DOC) still prevails as the most commonly found aluminum species (Merino et al., 1998). Fluoride $\left(\mathrm{F}^{-}\right)$, which strongly competes with DOC, was responsible for the strong complexation of $\mathrm{Al}$ in soil solution (Figure 2), given that the magnitude of such complexation, particularly in highly acidic soils $(\mathrm{pH}<4.8)$ is of fundamental importance, since it reduces the toxicity by the Al- $\mathrm{F}_{\mathrm{x}}$ species formation (Alva and Sumner, 1988).

Where the complexation of $\mathrm{Al}$ by $\mathrm{F}^{-}$is intense, it is possible that there has been an application of phosphate fertilizers containing fluoride (Zambrosi et al., 2007). In this study, the average content of $\mathrm{F}^{-}$in the samples was $0.24 \mathrm{mg} \mathrm{L}^{-1}$, well below that normally present in natural conditions, around $1 \mathrm{mg} \mathrm{L}^{-1}$ (Noemmik, 1953). Thus, aluminum complexes with phosphates and sulfates prevail over $\mathrm{Al}-\mathrm{F}^{\mathrm{x}}$ in samples with $\mathrm{F}^{-}$concentrations that are reduced or even absent, but are almost always less than the amounts of Al-DOC.

Sulfate $\left(\mathrm{S}^{-\mathrm{SO}_{4}}{ }^{2-}\right)$ and phosphate $\left(\mathrm{P}-\mathrm{Hx} \mathrm{PO}_{4 \mathrm{x}}\right)$ generally form ion pairs on smaller scales ("other species"), as observed by Zambrosi et al. (2008). In this study, the largest quantities of sulphate and phosphate appeared only in those samples in which there was no detectable fluoride ion or where either it was not detected at all or it was detected in very low concentrations (Figure 2 and 


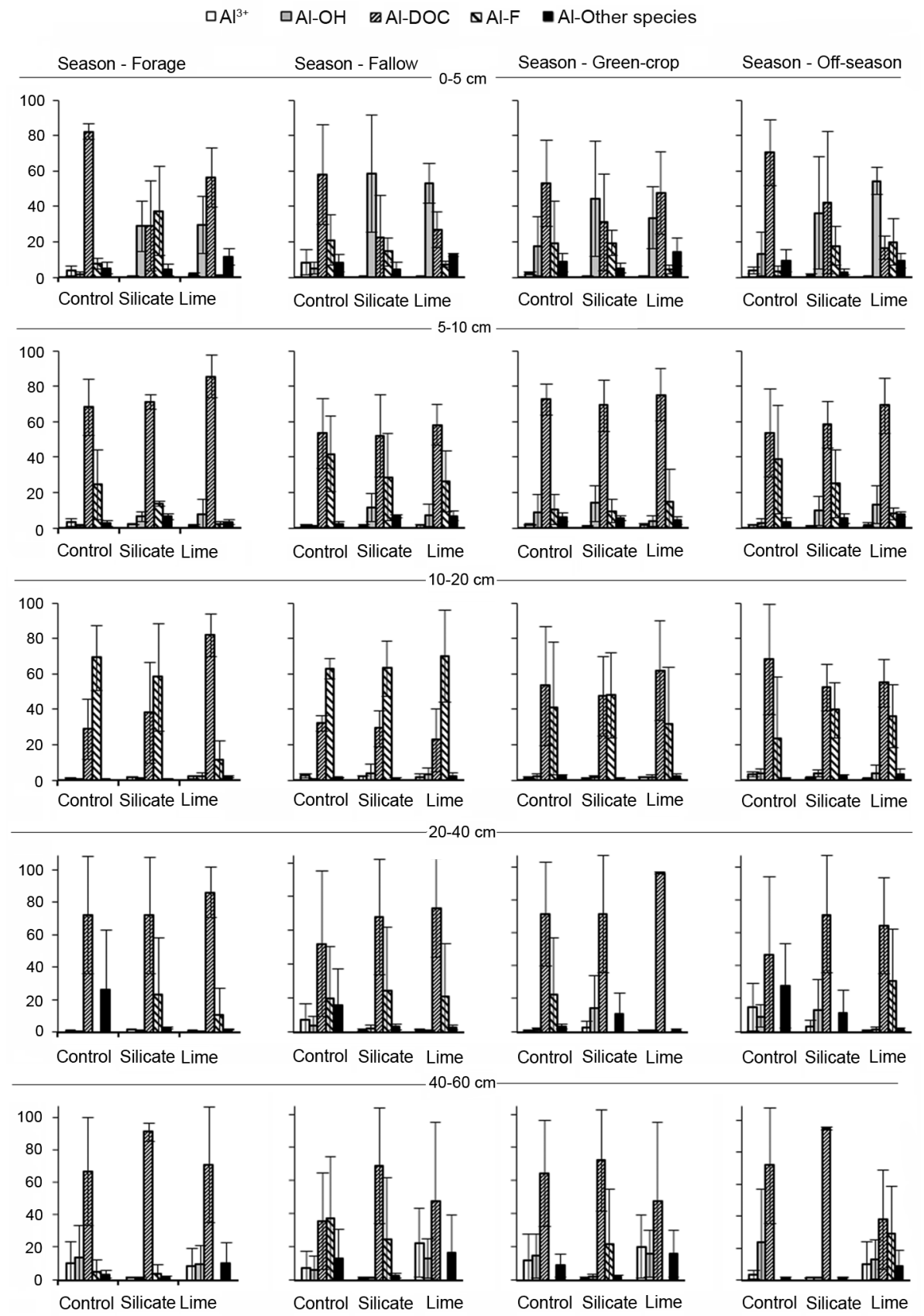

Figure 2 - Chemical species of aluminum (\%) in solution of an Oxisol under NT as a function of acidity amendments and crop systems. Vertical bars represent the mean deviation of replications for each treatment $(n=4)$. $\mathrm{Al}-\mathrm{OH}=\mathrm{Al}(\mathrm{OH})_{2}{ }^{+}+\mathrm{Al}(\mathrm{OH})^{2+}+\mathrm{Al}(\mathrm{OH})_{3}{ }^{0} ; \mathrm{Al}-\mathrm{F}=\mathrm{Al}(\mathrm{F})_{2}{ }^{+}+\mathrm{Al}(\mathrm{F})^{2+}+$ $\mathrm{Al}(\mathrm{F})_{3}{ }^{0}+\mathrm{Al}(\mathrm{F})_{4}{ }^{-}$. Other species: $\mathrm{AlHPO}_{4}{ }^{+}+\mathrm{Al}_{2}\left(\mathrm{PO}_{4}\right)^{3+}+\mathrm{AlSO}_{4}{ }^{+}+\mathrm{AlH}_{3} \mathrm{SiO}_{4}{ }^{2+}$.

Tables 3, 4, 5 and 6). Si complexation by $\mathrm{Al}$ and the formation of the $\mathrm{AlH}_{3} \mathrm{SiO}_{4}^{2+}$ species appeared in negligible quantities, in general, reaching a level less than $1 \%$ of all species of Al.

Sulfate and phosphate have similar binding constant values for complex formation with $\mathrm{Al}$, which are both very much lower than the binding constant values between $\mathrm{Al}$ and $\mathrm{F}$ (Walthall and Lindsay, 1996). These complexes follow a descending order of stability: $\mathrm{AlF}_{2}{ }^{+}$ $>\mathrm{AlH}_{2} \mathrm{PO}_{4}^{2+}>\mathrm{AlSO}_{4}{ }^{+}$with $\mathrm{K}_{0} \log$ values equal to 6.98, 3.50 and 3.10, respectively (Lindsay and Walthall, 1995). In many cases, the chemical species $\mathrm{Al}_{-} \mathrm{H}_{\mathrm{x}} \mathrm{PO}_{4 \mathrm{x}}(\mathrm{AlH}$ $\mathrm{PO}_{4}{ }^{+}+\mathrm{Al}_{2} \mathrm{PO}_{4}{ }^{3+}$ ) are barely detected (Zambrosi et al., 2008), which could also be due to the low concentration of phosphate ion in solution (Tables 3, 4, 5 and 6). In our study, sulfate also minimally influenced $\mathrm{Al}$ behav- 
ior in solution. According to Alvarez et al. (2002), the $\mathrm{AlSO}_{4}{ }^{+}$species, non-toxic to plants (Pavan et al., 1982), was rarely found in Spanish soils which have different parent materials. Zambrosi et al. (2008) reported small increases in participation of the $\mathrm{Al}-\mathrm{SO}_{4}{ }^{+}$species after the application of gypsum.

Liming and silicate application promoted an increase in the amount of hydroxylated forms of $\mathrm{Al}$ (Al$\mathrm{OH})$ due to the increased $\mathrm{pH}$ of the solution, especially in the first layer (Figure 2), and such forms are considered less toxic to plants than the free form $\mathrm{Al}^{+3}$. The range of variation in $\mathrm{pH}$ in this layer $(0.00-0.05 \mathrm{~m})$, taking into account the Season - Fallow system, was 4.8 for the samples of control treatment, 6.1 for samples that received lime and 6.4 in samples which received $\mathrm{Ca}-\mathrm{Mg}$ silicate (Table 6). In the same layer, under the Season - Green-crop system, the $\mathrm{pH}$ value was also lower for the control $(\mathrm{pH}=5.3)$ and higher for the silicates $(\mathrm{pH}$ $=6.1)$; already under the Season - Off-season system the control treatment also had the lowest $\mathrm{pH}$ (5.2), with the highest value being observed after the addition of lime $(\mathrm{pH}=6.3)$ (Tables 5 and 4 , respectively). Consequently, to what extent the application of lime and the calcium-magnesium silicates can neutralize the toxicity of $\mathrm{Al}$ is dependent on the $\mathrm{pH}$ of the soil solution as well as the presence of organic compounds and fluoride, and their respective concentrations and activities in solution. Among the species formed from $\mathrm{Al}$ in the soil solution, hydroxylated $\mathrm{Al}$ species are the most sensitive to management practices because of their dependence on the $\mathrm{pH}$ of the soil (Zambrosi et al., 2008).

\section{Calcium}

Regardless of sampling depth, crop system adopted and correctives reapplied, the free form of $\mathrm{Ca}$ $\left(\mathrm{Ca}^{2+}\right)$ was predominant in the soil solution, while the Ca-DOC species was in second place (Figure 3). This factor reflects the strong influence of OM, its subsequent decomposition, and the release of soluble organic compounds (DOC) in the speciation and complexation of $\mathrm{Ca}$ in soil solution. This complexation is important in soils that have accumulated OM on the surface due to management practices adopted, such as NT (Zambrosi et al., 2008).

The complexing of $\mathrm{Ca}$ by organic anions facilitates vertical mobility of this element deep in the soil, arising from the accumulation of organic residue and release of anions at the surface. These anions complex the cation $\left(\mathrm{Ca}^{2+}\right)$ and favor its leaching and mobilization (Cassiolato et al., 2000). There was considerable complexation of $\mathrm{Ca}$ by DOC up to a depth of $60 \mathrm{~cm}$ (Figure 3), and the same was observed by Zambrosi et al. (2008) up to a depth of $80 \mathrm{~cm}$. Thus, there is strong evidence that this form of leaching occurs in areas under NT.

Ionic pairs of calcium and inorganic anion (sulphate, chloride and nitrate) in the soil solution also collaborate in mobilizing this cation through the length of the soil profile (Pavan et al., 1984; Piirainem et al., 2002).
In this study, sulfate was the inorganic anion which most formed ionic pairs with calcium $\left(\mathrm{CaSO}_{4}\right)$ in all five layers of the soil sampled for all correctives and crop systems adopted. However, in general, the quantity did not exceed $5 \%$. There were also associations of Ca with fluoride $\left(\mathrm{CaF}^{+}\right)$, phosphate $\left.\left(\mathrm{CaHPO}_{4(\mathrm{aq})}\right) \mathrm{CaH}_{2} \mathrm{PO}_{4}^{+}\right)$, chlorine $\left(\mathrm{CaCl}^{+}\right)$, and nitrate $\left(\mathrm{CaNO}_{3}{ }^{+}\right)$("other species"), representing, however, when present, less than $1 \%$ of the total of all species of Ca. Adams (1971) states that the ion pairs of $\mathrm{Ca}^{2+}$ with these anions $\left(\mathrm{Cl}^{-}\right.$and $\left.\mathrm{NO}_{3}{ }^{-}\right)$do not occur or are very irrelevant, while the sulfate may have a higher expression compared to other inorganic anions, though still significantly less than DOC.

The results of $\mathrm{Ca}$ complexing with inorganic anions corroborate Chaves et al. (1991), who claim that the $\mathrm{NO}_{3}{ }^{-}, \mathrm{Cl}^{-}$and the $\mathrm{SO}_{4}{ }^{2-}$ are the main inorganic binders of $\mathrm{Ca}$ in solution, because they form part of the composition of the majority of fertilizers used in agriculture. $\mathrm{Ca}$ binding with inorganic anions follows the ensuing stability sequence: $\mathrm{SO}_{4}{ }^{2-}>\mathrm{Cl}^{-}>\mathrm{NO}_{3}{ }^{-}$, and the stability of each complex-dependent activity of anions in solution (Lindsay, 1979). The formation of these pairs of ions, in addition to acting directly on the mobility of calcium (leaching), interferes with availability to plants. These ionic pairings result in the chemical potential or real ion availability being reduced (Zambrosi et al., 2008).

Chaves et al. (1991) observed that the absorption of Ca by coffee (Coffea arabica) tree roots (after the application of calcium salts) in two northern acidic Oxisols in the state of Paraná, Brazil, followed the order: $\mathrm{Ca}^{2+}$ $>\mathrm{CaNO}_{3}{ }^{+}>\mathrm{CaCl}^{+}>\mathrm{CaSO}_{4}{ }^{0}$. Zambrosi et al. (2008) did not observe significant increases in the percentage of acidic soils in $\mathrm{CaSO}_{4}{ }^{0}$ even after gypsum application, and the $\mathrm{CaSO}_{4}{ }^{0}$ was uniformly distributed along the profile (up to $80 \mathrm{~cm}$ ) regardless of application of lime or gypsum. Liming and silicate application had no obvious effects on the distribution of the species of calcium, and there were no noticeable changes in comparisons made between depths. Unlike in Zambrosi et al. (2007), free form $\mathrm{Ca}\left(\mathrm{Ca}^{2+}\right)$ did not decrease at lower depths in the soil profile, but maintained their high activity level and a positive correlation with the total concentrations of $\mathrm{Ca}$ in the aqueous extract (Table 2 and Figure 4). On average, the activity of calcium $\left(a \mathrm{Ca}^{2+}\right)$ corresponded to $65 \%$ of Cat, in the five layers studied (Table 2 and Figure 4).

As there were no differences between the applications of lime and $\mathrm{Ca}$ and $\mathrm{Mg}$ silicates in any of the five layers, it can be inferred that the positive correlation between the activity of the free forms of $\mathrm{Ca}\left(a \mathrm{Ca}^{2+}\right)$ and the total concentrations of this element in solution (Cat) were due to increased $a \mathrm{Ca}^{2+}$ as a consequence of greater Cat concentrations in solution, which are represented by the higher percentages obtained with the free form of $\mathrm{Ca}^{2+}$ (Table 2 and Figure 3). Zambrosi et al. (2007) highlighted increases in soluble concentrations of $\mathrm{Ca}$ and their respective activities in the free form up to $80 \mathrm{~cm}$, emphasizing the importance of underground enrichment in terms of Ca supply to the plant root system. 


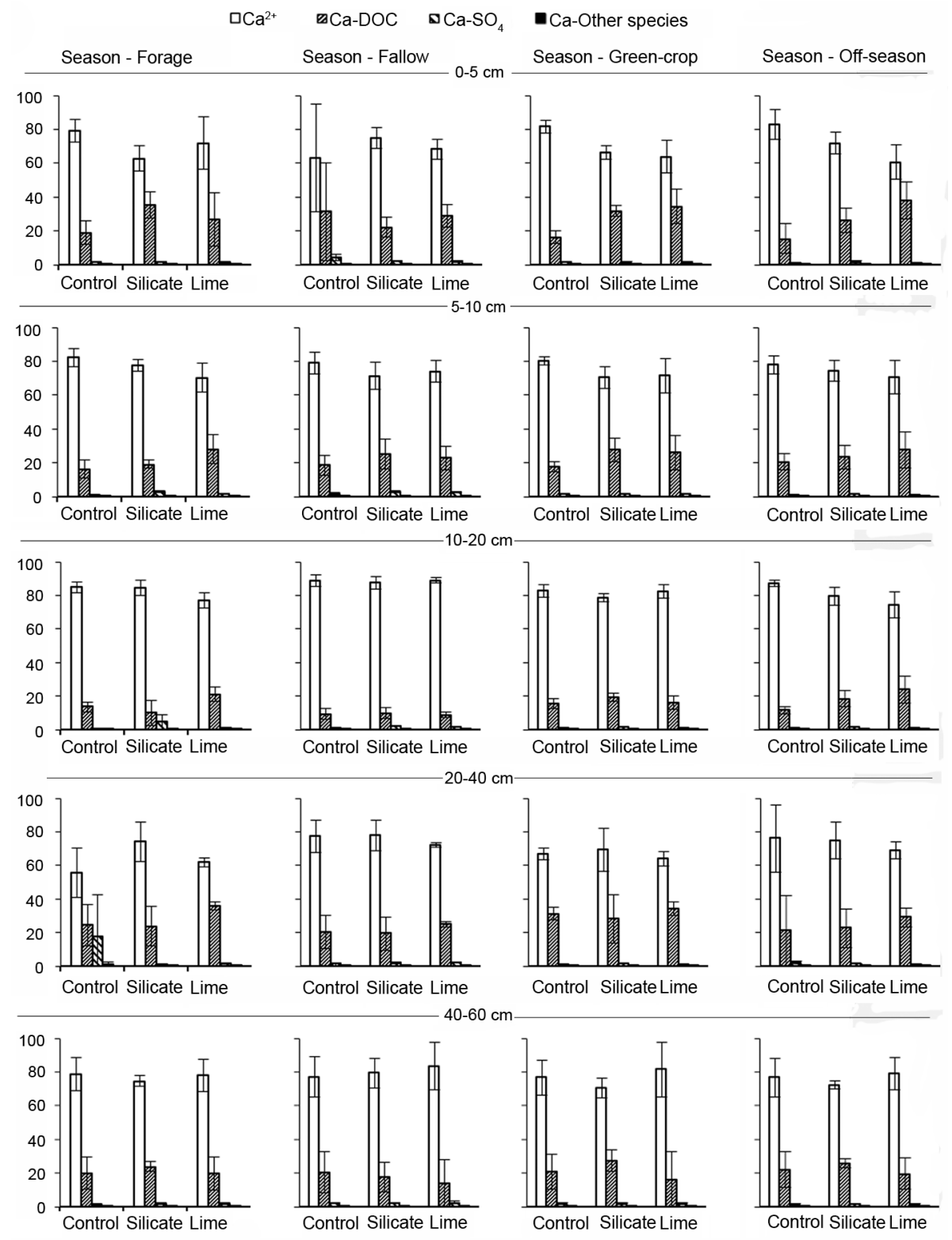

Figure 3 - Chemical species of calcium (\%) in solution of an Oxisol under NT as a function of acidity amendments and crop systems. Vertical bars represent the mean deviation of replications for each treatment $(n=4)$. Other species: $\mathrm{CaCl}^{+} ; \mathrm{CaNO}_{3}^{+} ; \mathrm{CaF}^{+} ; \mathrm{CaHPO}_{4}(\mathrm{aq}) ; \mathrm{CaH}_{2} \mathrm{PO}_{4}^{-}$.

\section{Magnesium}

As with $\mathrm{Ca}$, DOC was the main $\mathrm{Mg}$ binder in the soil solution (Mg-DOC), but to a lesser extent than the Ca-DOC. The free forms $\left(\mathrm{Mg}^{2+}\right)$ were predominant and in even greater quantities than $\mathrm{Ca}^{2+}$ (Figure 5). In general, among the inorganic anions, the interaction with sulphate $\left(\mathrm{MgSO}_{4}{ }^{\circ}\right)$ occurred in quantities close to $3 \%$, or less than the $\mathrm{CaSO}_{4}{ }^{0}$, where up to $5 \%$ for these species was recorded. The only exceptions were found in the layer of 20-40 cm under the Season - Forage system and control treatment, where approximately $15 \%$ of $\mathrm{MgSO}_{4}$ was observed, and the same applies to the $\mathrm{Ca}-\mathrm{SO}_{4}$ (Figures 5 and 3, respectively). Zambrosi et al. (2008) ob- served that the ion pair $\mathrm{MgSO}_{4}$ represented at most $1 \%$ of the total $\mathrm{Mg}$ species in solution of an Oxisol from the state of Paraná.

The inorganic anion most responsible for the mobility of $\mathrm{Mg}$ is sulfate $\left(\mathrm{SO}_{4}{ }^{2-}\right)$, which explains the intense leaching of $\mathrm{Mg}$ when gypsum is applied. The $\mathrm{MgSO}_{4}{ }^{0}$ complex contributes significantly to the total concentration of $\mathrm{Mg}^{2+}$ in solution when the concentration of $\mathrm{SO}_{4}^{-2}$ is above $10^{-4} \mathrm{~mol} \mathrm{~L}^{-1}$ (Lindsay, 1979). The forms of $\mathrm{Mg}$ with other inorganic anions, such as fluoride $\left(\mathrm{MgF}^{+}\right)$, phosphate $\left(\mathrm{MgHPO}_{4(\text { aq })}\right)$, nitrate $\left(\mathrm{MgNO}_{3}{ }^{+}\right)$and chloride $\left(\mathrm{MgCl}^{+}\right)$were detected in amounts close to $0 \%$ of the 

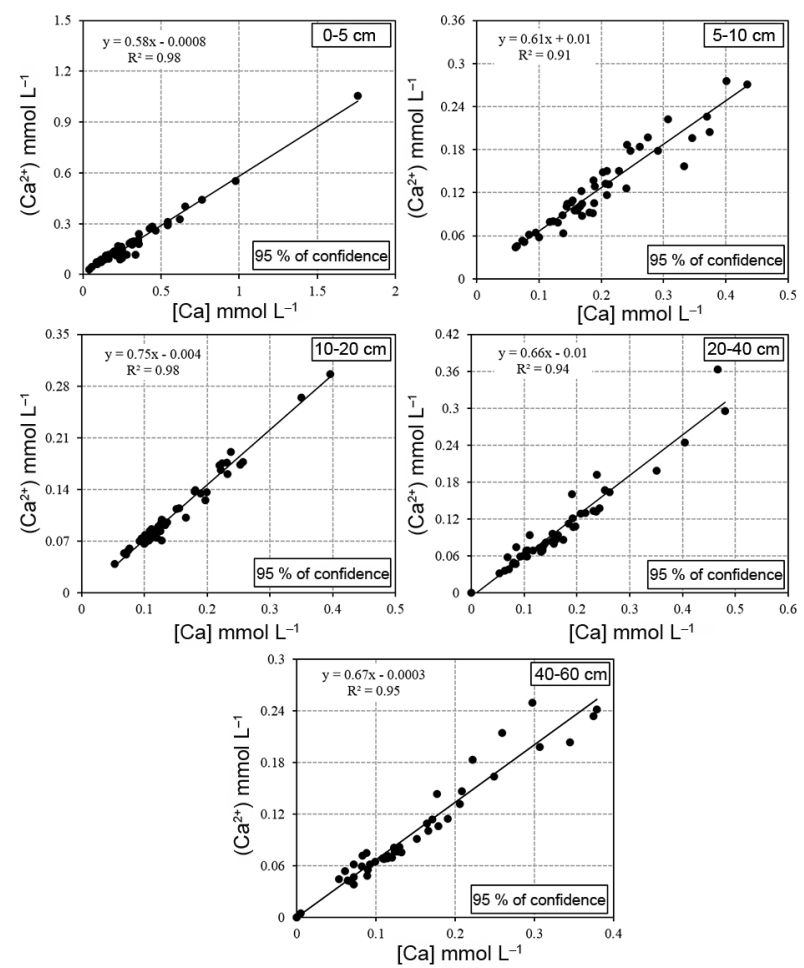

Figure 4 - Linear regressions for calcium concentration ([Ca]) and the activity of its free form $\left(\left(\mathrm{Ca}^{2+}\right)\right)$ in solution at five depths of an Oxisol under NT.

total ("other species") (Figure 5). The magnesium complexes of chloride and nitrate have stability constants which are very close $\left(\log \mathrm{K}_{0} \sim 0.6\right)$, but are of minimal significance in solution (Lindsay, 1979).

The amount of free form magnesium $\left(\mathrm{Mg}^{2+}\right)$ is much greater than when in association with organic anions and, in the main, inorganic anions (Table 2 and Figure 6). For the inorganic anions, the nitrate ion pair also failed to form an ionic pair in significant amounts with $\mathrm{Mg}$, which can indicate little activity of this cation at depth. The organic anions were significant contributors to the mobility of $\mathrm{Mg}$, as were the components of the soil solution which combined with a higher proportion of this cation across all layers, as observed by Zambrosi et al. (2008). The total concentrations of $\mathrm{Mg}$ in aqueous extract $(\mathrm{Mg} t)$ were highly positively correlated with $a \mathrm{Mg}^{2+}$ in all layers (Table 2 and Figure 6). On average, $a \mathrm{Mg}^{2+}$ corresponded to $81 \%$ of the total $\mathrm{Mg}$ concentration in the five layers studied, or even higher than the percentage of $a \mathrm{Ca}^{2+}$ compared to Cat. This fact can be explained by higher percentages of $\mathrm{Mg}^{2+}$ and lower $\mathrm{Mg}$-DOC compared to $\mathrm{Ca}^{2+}$ and $\mathrm{Ca}-\mathrm{DOC}$, respectively.

\section{Potassium}

The activity of free form $\mathrm{K}\left(a \mathrm{~K}^{+}\right)$was not affected by any of the treatments (Table 2 and Figure 7), even in the two deepest layers, where total $\mathrm{K}$ concentrations were reduced (Tables 3, 4, 5 and 6). The activity of $\mathrm{K}$ free ions positively correlated with soluble concentrations (Table 2 and Figure 7), and high $a \mathrm{~K}^{+}$corresponded to total concentrations of the ion in solution $(\mathrm{K} t)$. On average, $a \mathrm{~K}^{+}$corresponded to $96 \%$ of the total concentration of $\mathrm{K}$ in solution for all depths, this number being quite similar to that observed by Zambrosi et al. (2007), who observed a value of $95 \%$ for such activity. Potassium, unlike $\mathrm{Ca}$ and $\mathrm{Mg}$, was not complexed by organic anions in solution. The percentages of ion pairs to $\mathrm{K}\left(\mathrm{KCl}_{(\mathrm{aq})}\right.$ $\mathrm{KNO}_{3}$ and $\mathrm{KSO}_{4}{ }^{-}$) represented less than $1 \%$ of the total, regardless of crop system, treatment or depth sampled.

In well-drained soils, complexes with $\mathrm{K}$ have little relevance (Lindsay, 1979). In moist tropical soils with high clay content, iron oxides form strong aggregates in Oxisols which allow leaching and facilitate drainage. However, the interaction of potassium with anions in soil solution affects the leaching of the monovalent cationic form $\left(\mathrm{K}^{+}\right)$since potassium facilitates the migration of anions along the soil profile.

\section{Silicon}

Monosilicic acid $\left(\mathrm{H}_{4} \mathrm{SiO}_{4}\right)$ prevailed in all the soil layers for all crop systems, regardless of the corrective applied (Figure 8), which was expected in Oxisols judging by the range of variation in $\mathrm{pH}$ observed in water extracts. The $\mathrm{H}_{4} \mathrm{SiO}_{4}$ species, in general, accounted for almost $100 \%$ of Si species in soil solution, with a number of exceptions (Figure 8). The activity of the most protonated free form of $\mathrm{Si}$ in the water sample taken from the ground $\left(a \mathrm{H}_{4} \mathrm{SiO}_{4}\right)$ also remained unaffected by treatments (Table 2 and Figure 9), even in the deepest layers, where the concentrations of $\mathrm{H}_{4} \mathrm{SiO}_{4}$ were reduced (Tables 3, 4, 5 and 6).

The $\mathrm{H}_{4} \mathrm{SiO}_{4}$ activity positively correlates with the soluble concentration of the species (Table 2 and Figure 9). The high level of $a \mathrm{H}_{4} \mathrm{SiO}_{4}$ corresponded to the total concentrations of the species in solution $\left(\mathrm{H}_{4} \mathrm{SiO}_{4} t\right)$, which further enhances the strong presence of monosilicic acid in soil solution. On average, $\mathrm{aH}_{4} \mathrm{SiO}_{4}$ corresponded to almost $100 \%$ of $\mathrm{H}_{4} \mathrm{SiO}_{4}$ t solution.

At $\mathrm{pH}$ values around 7, less than $1 \%$ in the form of $\mathrm{H}_{4} \mathrm{SiO}_{4}$ ionizes to negative (silicate anion $\mathrm{H}_{3} \mathrm{SiO}_{4}{ }^{-}$), and the degree of ionization decreases as the $\mathrm{pH}$ decreases (McKeague and Cline, 1963). For the conditions of this study, we noticed the substantially reduced presence by approximately, in general, less than $1 \%$ of the aluminum species complexed with silicate anions $\left(\mathrm{AlH}_{3} \mathrm{SiO}_{4}{ }^{2+}\right)$, which could be responsible for a decrease in $\mathrm{Al}^{3+}$ phytoxicity in solution. Where the $\mathrm{pH}$ values exceeds the neutral level, the presence of silicate in solution increased as $\mathrm{pH}$ increased. However, other complex aluminum ion stability constants have even greater stability than $\mathrm{H}_{3} \mathrm{SiO}_{4}{ }^{-}$and start to predominate in solution as is the case with $\mathrm{Al}$ complexes formed with hydroxyls (Figure 10).

On the other hand in a solution with the presence of only aluminum and silicon in equimolar fractions, it is known that the $\mathrm{AlH}_{3} \mathrm{SiO}_{4}^{2+}$ species is present in solution 


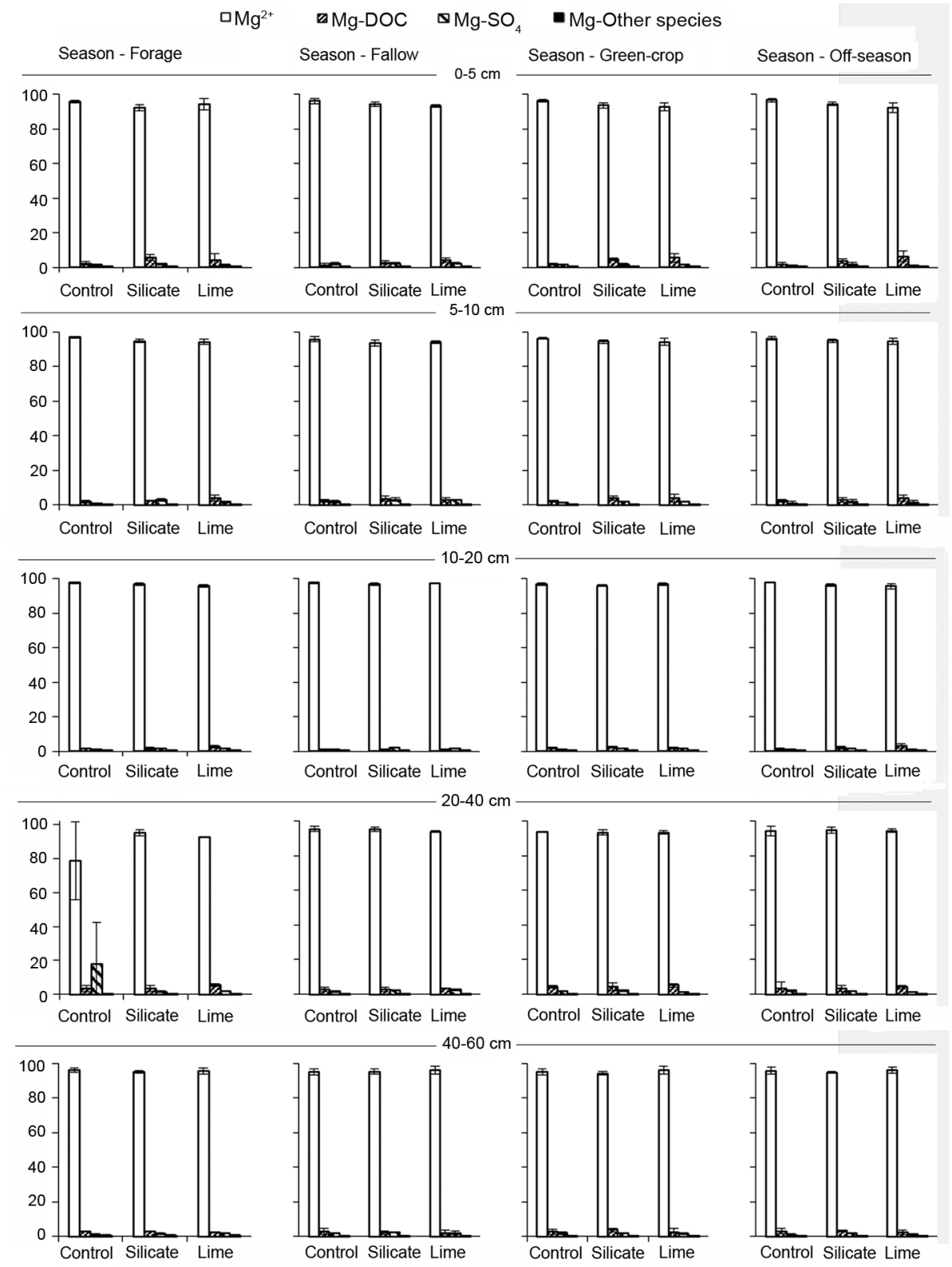

Figure 5 - Chemical species of magnesium (\%) in solution of an Oxisol under NT as a function of acidity amendments and crop systems. Vertical bars represent the mean deviation of replications for each treatment $(\mathrm{n}=4)$; Other species: $\mathrm{MgCl}^{+} ; \mathrm{MgNO}_{3}^{+} ; \mathrm{MgF}^{+} ; \mathrm{MgHPO}_{4(\text { aq)})}$.

in $\mathrm{pH}$ ranges varying from 3.5 to 6.5 , such as is commonly found in humid tropical soils, and its percentage in solution can reach approximately $10 \%$ of the distribution of species at $\mathrm{pH}$ values close to 5.5 (Figure 10). The activity of these species reached levels comparable to those in their solution concentrations. The presence of more ions in solution alters the distribution of all possible species in solution depending on their concentration and respective activities.

The aluminosilicate species $\left(\mathrm{AlH}_{3} \mathrm{SiO}_{4}{ }^{2+}\right)$ in solution represented approximately $2 \%$ under the Season - Forage system, in the 0.00-0.05 m layer where lime was applied to the surface (Figure 8). In this sample, the $\mathrm{pH}$ value (24 months after the last application of lime) was 5.7 (Table 3), and aluminum and silicon concentrations were 0.39 and $0.36 \mathrm{mmol} \mathrm{L}^{-1}$, respectively (Table $3)$, which is considerably higher compared to other treatments and other production systems in the five layers (Tables 3, 4, 5 and 6). Thus, high concentrations may also have influenced the activity of these ions in solution and consequently the species formed, and behavior patterns were similar to those shown in synthetic solutions (Figures 8 and 11). For the value of the $\mathrm{pH}$ analyzed (5.7), $\mathrm{Al}^{3+}$ is still present in solution in quantities sufficient 

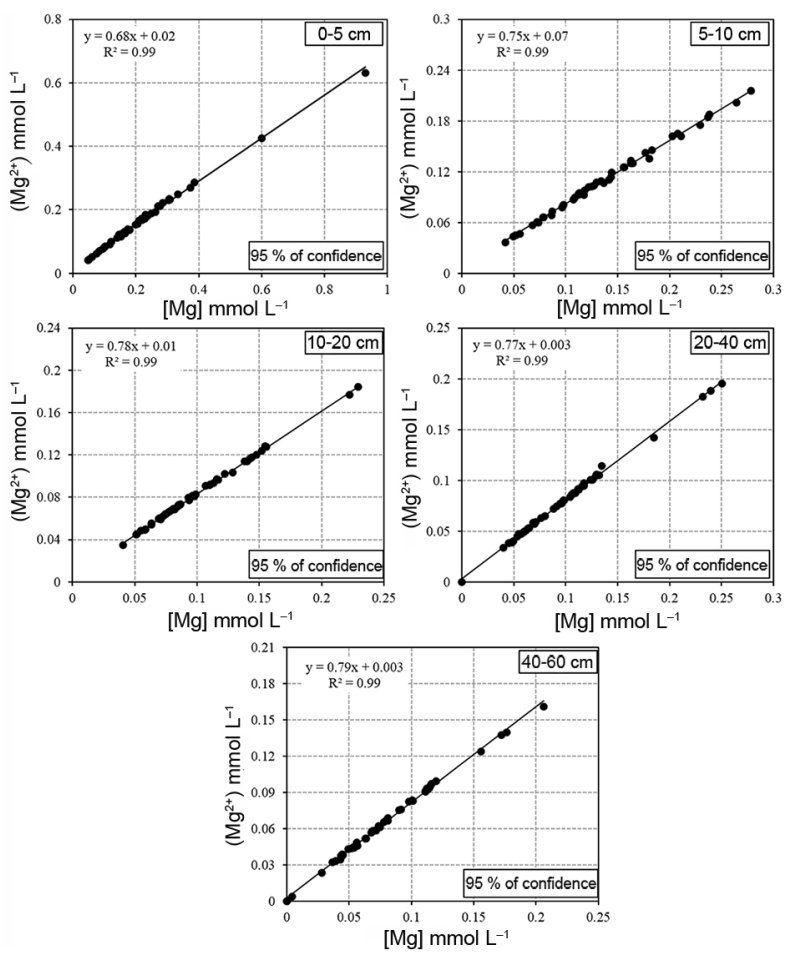

Figure 6 - Linear regressions for magnesium concentration ([Mg]) and the activity of its free form $\left(\left(\mathrm{Mg}^{2+}\right)\right)$ in solution at five depths of an Oxisol under NT.

to form the ion pair $\mathrm{AlH}_{3} \mathrm{SiO}_{4}{ }^{2+}$, whereas the solubility of free form $\mathrm{Al}^{3+}$ is nil after $\mathrm{pH}=6$ and the silicate anion begins to increase when the $\mathrm{pH}$ is around 7 (neutral range) (Figure 10).

The $\mathrm{Si}\left(\mathrm{Si}^{4+}\right)$ species complexed with the fluoride ion $\left(\mathrm{F}^{-}\right)$was also detected, but in concentrations close to zero, despite having a relatively high stability constant $\left(\mathrm{SiF}_{6}{ }^{2-}, \log \mathrm{K}_{0}=30.18\right)$. This is because the free $\mathrm{Si}^{4+}$ species hardly appears in the tropical acid soil solution due to the low $\mathrm{pH}$ value. The solubility of $\mathrm{Si}^{4+}$ occurs in an alkaline environment, and even this free form is minimally complexed by fluoride $\left(\mathrm{F}^{-}\right)$.

\section{Sulfur}

Ionic pairs of monosilicic acid with sulfate were formed in solution $\left(\mathrm{H}_{4} \mathrm{SiO}_{4}-\mathrm{SO}_{4}{ }^{2-}\right)$, as was observed for $\mathrm{Ca}$ and $\mathrm{Mg}$ under the Season - Forage system (Figures 3 and 5) in larger amounts, and Season - Fallow for Si in the $20-40 \mathrm{~cm}$ and $5-10 \mathrm{~cm}$ layers, respectively, in lesser quantities (Figure 8). The average concentration of $\mathrm{S}$ was $142.5 \mathrm{mmol} \mathrm{L}^{-1}$ (or $4,570 \mathrm{mg} \mathrm{dm}^{-3}$ ) for the Season - Forage system, in the control treatment at a depth of 20-40 $\mathrm{cm}$ (Table 3); this amount is approximately 4,500 times the concentration of silicon $\left(\mathrm{Si}=0.04 \mathrm{mmol} \mathrm{L}^{-1}\right.$ or 1.02 $\mathrm{mg} \mathrm{dm^{-3 }}$ ) for the same sample, that is, an $S_{\text {total }}$ value certainly much higher than that normally found in soils, which indicates probable contamination of the area for the sample in question. Neptune et al. (1975) classified
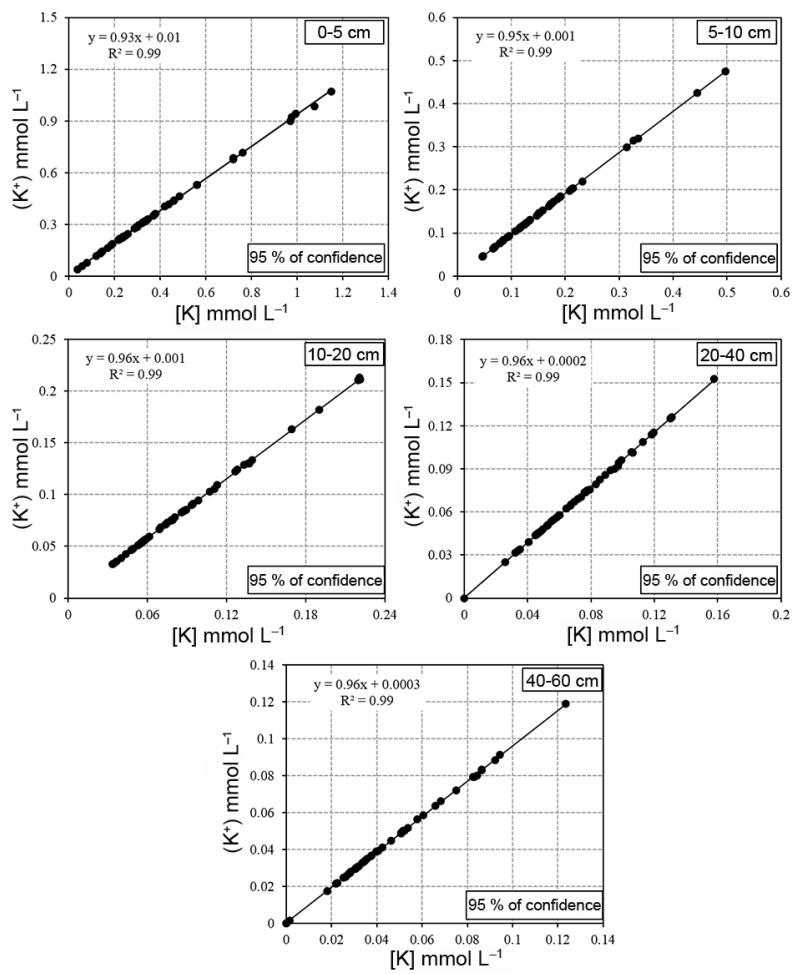

Figure 7 - Linear regressions for potassium concentration ([K]) and the activity of its free form $\left(\left(\mathrm{K}^{+}\right)\right)$in solution at five depths of an Oxisol under NT.

as average the $S_{\text {total }}$ content of between 209 and $398 \mathrm{mg}$ $\mathrm{kg}^{-1}$ of a tropical soil after alkaline digestion with $\mathrm{NaO}$ Br. Sulfur contents in the humid tropical soils would be even lower than those in a temperate climate due to sulfate leaching along the length of the soil profile.

Sulfate $\left(\mathrm{SO}_{4}{ }^{2-}\right)$ was the predominant species, occupying approximately $95 \%$ of the total in soils. Moreover, the average total percentage taken from all depths was $82 \%$ for sulfate activity $\left(a \mathrm{SO}_{4}{ }^{2-}\right)$ with respect to the $\mathrm{S}_{\text {total }}(\mathrm{S} t)$ obtained in water extracts and the coefficient of determination was 0.99 . For the deepest two layers, the percentage ratio of $a \mathrm{SO}_{4}{ }^{2-} / \mathrm{S} t$ was $82 \%$, and the $\mathrm{R}^{2}=1$; and $85 \%$ with $\mathrm{R}^{2}=0.99$ to $20-40$ and 40-60 $\mathrm{cm}$, respectively (for all the results, $p<0.05$ ). Under conditions of synthetic solution, without interference of the activity of other ions in solution, the presence of $\mathrm{Si}$ species complexed with $\mathrm{SO}_{4}{ }^{2-}$, defined by the ion-pair $\mathrm{H}_{4} \mathrm{SiO}_{4}-\mathrm{SO}_{4}^{2-}, \log \mathrm{K}_{0}=-0.54$ (Visual MINTEQ) is registered in percentages above $25 \%$ for concentrations of $\mathrm{S}$ thousands of times higher than $\mathrm{Si}$ (Figure 12).

These factors were reflected in the formation of the $\mathrm{H}_{4} \mathrm{SiO}_{4}-\mathrm{SO}_{4}{ }^{2-}$ species, in relation to other species, and were different from the $\mathrm{H}_{4} \mathrm{SiO}_{4}$ species, even in the presence of other ions in solution (Figure 8). The application of gypsum (post-liming), as well as the adoption of $\mathrm{NT}$, can increase $\mathrm{SO}_{4}^{2-}$ content in the soil. Firstly, to promote mobility of $\mathrm{SO}_{4}^{2-}$ down to the deepest layers 


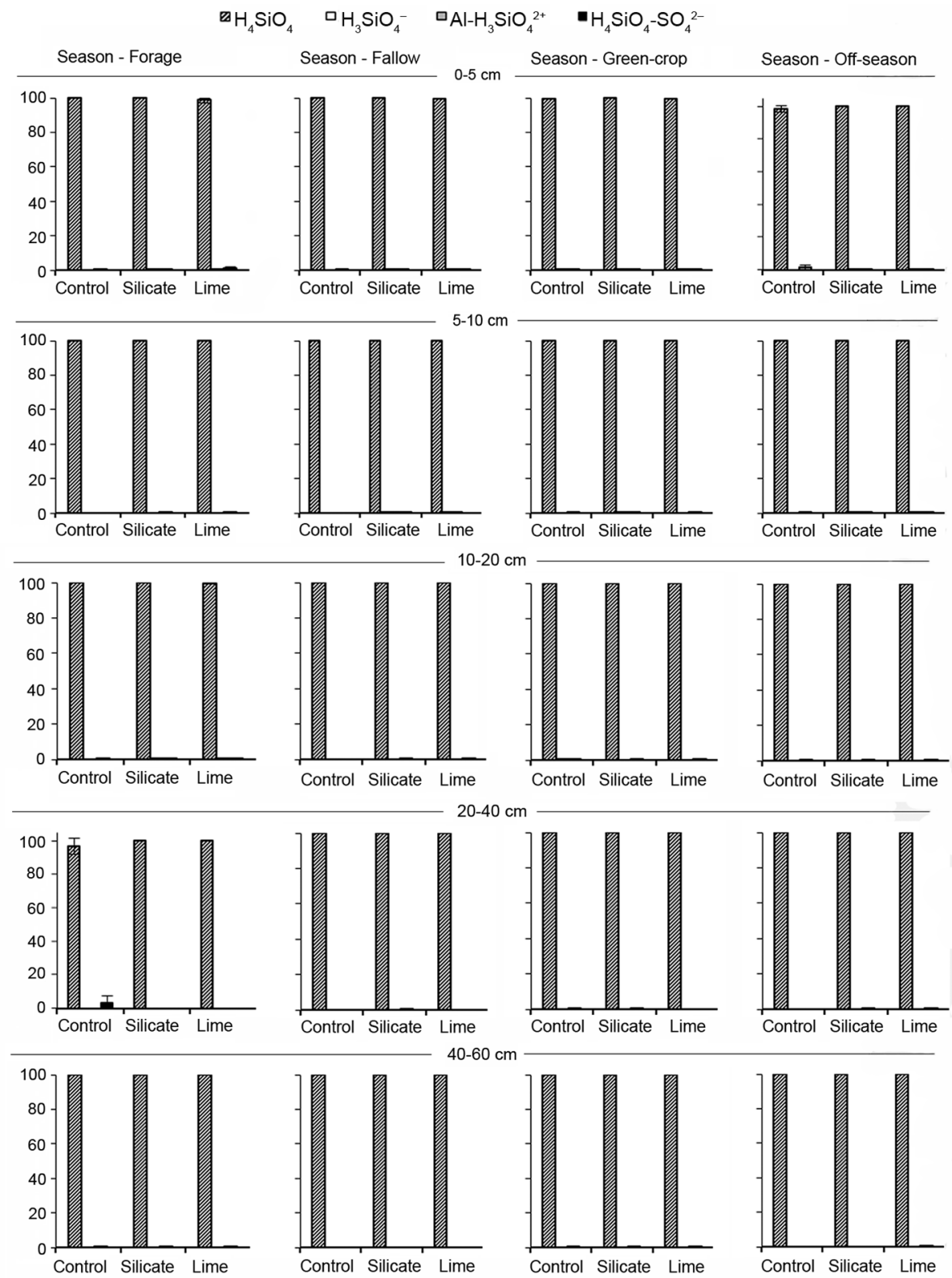

Figure 8 - Chemical species of silicon (\%) in solution of an Oxisol under NT as a function of acidity amendments and crop systems; Vertical bars represent the mean deviation of replications for each treatment $(n=4)$.

(where a good part of the roots is located) and, therefore, complex toxic elements and has other components in solution and secondly, to ensure S organic mineralization, responsible for more than $90 \%$ of the total $\mathrm{S}$ in soils (Neptune et al., 1975).

\section{Conclusions}

For all crop systems, silicon was almost entirely in the form of $\mathrm{H}_{4} \mathrm{SiO}_{4}$ in the aqueous extract, and the activity of this species was equivalent to its concentration in solution, providing negligible percentage values of both species $\mathrm{H}_{3} \mathrm{SiO}_{4}{ }^{-}$and $\mathrm{Al}-\mathrm{H}_{3} \mathrm{SiO}_{4}{ }^{2+}$, regardless of depth sampled and the amendment applied.

Unlike $\mathrm{K}, \mathrm{Ca}$ and $\mathrm{Mg}$ formed complexes with the DOC no matter the sampling depth, and the free forms of these three cations are the most prevalent species in this experiment. Their total concentrations were also strongly correlated with the activity of free forms in solution.

Aluminum was predominantly complexed with dissolved organic carbon and not in its free form $\mathrm{Al}^{3+}$ in aqueous extract, as expected, this result being observed even at the lowest depths $(60 \mathrm{~cm})$. 

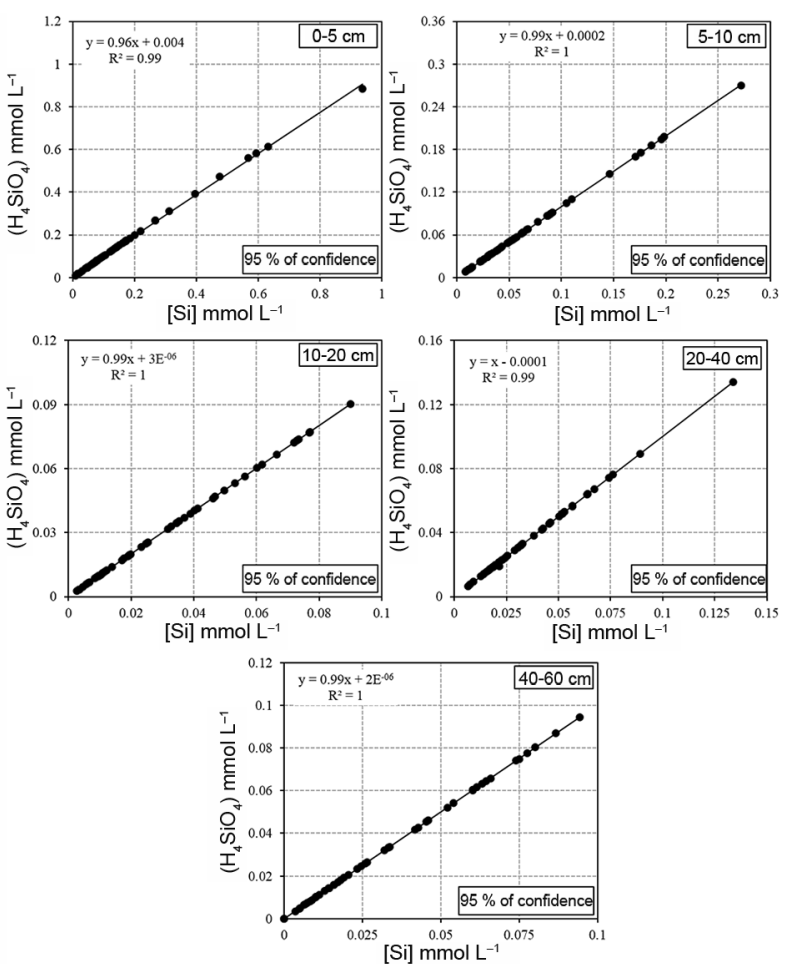

Figure 9 - Linear regressions for silicon concentration ([Si]) and the activity of its silicic acid form $\left(\left(\mathrm{H}_{4} \mathrm{SiO}_{4}\right)\right)$ in solution at five depths of an Oxisol under NT.

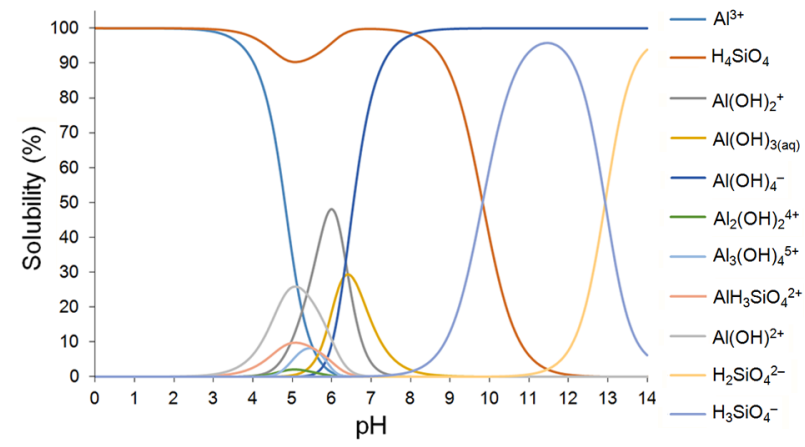

Figure 10 - Distribution of species and solubility of silicon and aluminum as a function of $\mathrm{pH}$; Figure made by Visual MINTEQ software (Gustafsson, 2013).

\section{Acknowledgments}

To FAPESP (São Paulo Research Foundation) for financial support of this research (Registry numbers: 2011/10566-6, 2013/18594-4, 2013/18694-9, 2013/02000-8 and 2014/08768-8) and CNPq (Brazilian National Council for Scientific and Technological Development) for productivity grants in research to Luís Reynaldo Ferracciú Alleoni and Carlos Alexandre Costa Crusciol.

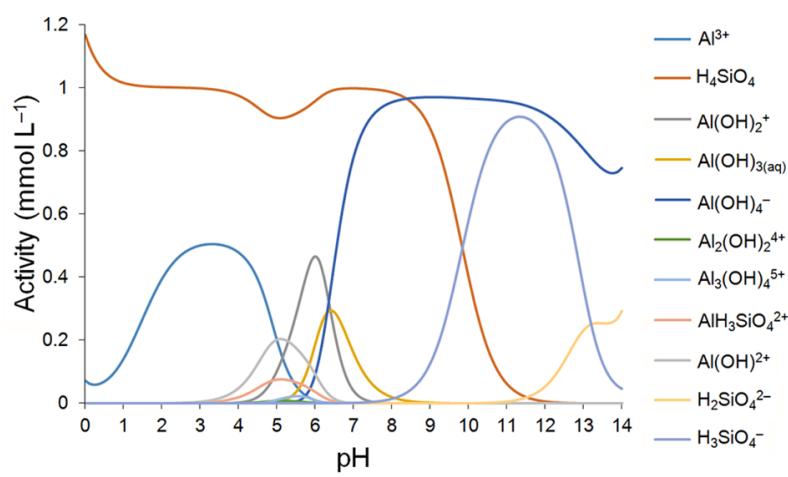

Figure 11 - Activity of silicon and aluminum species as a function of pH; Figure made by Visual MINTEQ software (Gustafsson, 2013).

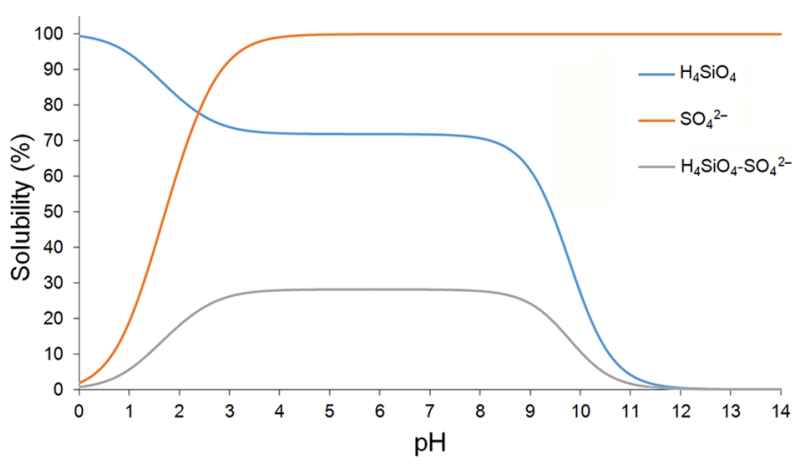

Figure 12 - Distribution of silicon species $\left(\mathrm{H}_{4} \mathrm{SiO}_{4}\right)$ and sulphate $\left(\mathrm{SO}_{4}{ }^{2-}\right)$, ratio $\mathrm{S} / \mathrm{Si}=4,500\left(0.9 / 0.0002 \mathrm{~mol} \mathrm{~L}^{-1}\right)$, as a function of $\mathrm{pH}$; Figure made by Visual MINTEQ software (Gustafsson, 2013).

\section{References}

Adams, F. 1971. Ionic concentrations and activities in soil solutions. Soil Science Society of America Proceedings 35: 420-426.

Adams, M.L.; McIntosh, P.D.; Patterson, R.D.; Powell, K.J. 1999. Aluminium speciation in seasonally dry high country soils, South Island, New Zealand. Soil Research 37: 1005-1015.

Alleoni, L.R.F.; Cambri, M.A.; Caires, E.F.; Garbuio, F.J. 2010. Acidity and aluminum speciation as affected by surface liming in tropical no-till soils. Soil Science Society of America Journal 74: 1010-1017.

Alva, A.K.; Sumner, M.E. 1988. Effects of phosphogypsum or calcium sulfate on aluminum reactive aluminum in solutions at varying $\mathrm{pH}$. Communications in Soil Science and Plant Analysis 19: $1715-1730$.

Álvarez, E.; Monterroso, C.; Fernándes Marcos, M.L. 2002. Aluminium fractionation in Galicia (NW Spain) forest soils as related to vegetation and parent material. Forest Ecology and Management 166: 193-206.

Bessho, T.; Bell, L.C. 1992. Soil solid and solution phase changes and mung bean response during amelioration of aluminium toxicity with organic matter. Plant and Soil 140: 193-192.

Cassiolato, M.E.; Meda, A.R.; Pavan, M.A.; Miyazawa, M.; Oliveira, J.C. 2000. Evaluation of oat extracts on the efficiency of lime in soil. Brazilian Archives of Biology and Technology 43: 533-536. 
Chaves, J.C.D.; Pavan, M.A.; Miyazawa, M. 1991. Chemical speciation of soil solution to assess calcium and aluminum uptake by coffee roots. Pesquisa Agropecuária Brasileira 26: 447-453 (in Portuguese, with abstract in English).

Datnoff, L.E.; Rodrigues, F.A.; Seebold, K.W. 2007. Silicon and plant disease. p. 233-246. In: Elmer, W.H.; Huber, D.M.; Datnoff, L.E., eds. Mineral nutrition and plant disease. The American Phytopathological Society, St Paul, MN, USA.

Datnoff, L.E.; Snyder, G.H.; Korndorfer, G.H. 2001. Silicon in agriculture. Elsevier Science, Amsterdam, The Netherlands.

Drabeck, O.; Mladkoba, L.; Boruvka, L.; Szakova, J.; Nikodem, A.; Nemecek, K. 2005. Comparison of water-soluble and exchangeable forms of $\mathrm{Al}$ in acid forest soils. Journal of Inorganic Biochemistry 99: 1788-1795.

Fox, R.L.; Silva, J.A. 1978. Symptoms of plant malnutrition silicon an ergonomically essential nutrient for sugarcane. University of Hawaii, Manoa, HI, USA. p. 85.

Grimm, D.M.; Azarraga, L.V.; Carreira, L.A.; Susetyo, W. 1991. Continuous multiligand distribution model used to predict the stability constant of cooper (II) metal complexation with humic material from fluorescence quenching data. Environmental Science \& Technology 25: 1427-1431.

Gustafsson, J.P. 2013. Visual MINTEQ. Available at: http:// vminteq.lwr.kth.se/ [Accessed May 20, 2013]

Jones, L.H.P.; Handreck, K.A. 1967. Silica in soils, plants, and animals. Advances in Agronomy 19: 107-149.

Hiradate, S. 2011. Speciation of aluminum in soil environments. Soil Science and Plant Nutrition 50: 303-314.

Hobara, S.; Fukunaga-Yoshida, S.; Suzuki, T.; Matsumoto, S.; Matoh, T.; Ae, N. 2016. Plant silicon uptake increases active aluminum minerals in root-zone soil: implications for plant influence on soil carbon. Geoderma 279: 45-52.

Lindsay, W.L. 1979. Chemical Equilibria in Soils. John Wiley, New York, NY, USA. p. 450.

Lindsay, W.L.; Walthall, P.M. 1995. The solubility of aluminum in soils. p. 333-361. In: Sposito, G., ed. The environmental chemistry of aluminum. Lewis, Boca Raton, FL, USA.

Lindsay, W.L.; Walthall, P.M. 1996. The chemistry of aluminum in surface waters. p. 363-418. In: Sposito, G., ed. The environmental chemistry of aluminum. 2ed. CRC Press, Boca Raton, FL, USA.

Ma, J.F.; Furukawa, J. 2003. Recent progress in the research of external $\mathrm{Al}$ detoxification in higher plants: a mini-review. Journal of Inorganic Biochemistry 97: 46-51.

McKeague, J.A.; Cline, M.G. 1963. Silica in soil solutions. II. The adsorption of monosilicic acid by other substances. Canadian Journal of Soil Science 43: 83-95.
Merino, A.; Macías, F.; García-Rodeja, E. 1998. Aluminum dynamics in experimental acidified soils from a humidtemperate region of south Europe. Chemosphere 36: 11371142.

Neptune, A.M.L.; Tabatabai, M.A.; Hanway, J.J. 1975. Sulfur fractions and carbon nitrogen-phosphorus relationships in some Brazilian and Iowa soils. Soil Science Society of America Proceedings 39: 51-55.

Noemmik, H. 1953. Fluorine in Swedish agricultural products, soil and drinking water. Acta Polytechnica 127: 1-121.

Pavan, M.A.; Bingham, F.T.; Pratt, P.F. 1982. Toxicity of Al to coffee in Ultisols and Oxisols amended with $\mathrm{CaCO}_{3}, \mathrm{MgCO}_{3^{\prime}}$ and $\mathrm{CaSO}_{4} \cdot 2 \mathrm{H}_{2} \mathrm{O}$. Soil Science Society of America Journal 46: 1201-1207.

Pavan, M.A.; Bingham, F.T.; Pratt, P.F. 1984. Redistribution of exchangeable calcium, magnesium, and aluminum following lime or gypsum applications to a Brazilian Oxisol. Soil Science Society of America Journal 48: 33-38.

Piirainem, S.; Finér, L.; Starr, M. 2002. Deposition and leaching of sulphate and base cations in mixed boreal forest in eastern Finland. Water, Air and Soil Pollution 131: 185-204.

Raij, B. 1998. Bioavailable tests: alternatives to standard soil extractions. Communications in Soil Science and Plant Analysis 29: 1553-1570.

Ramos, L.A.; Nolla, A.; Korndörfer, G.H.; Pereira, H.S.; Camargo, M.D. 2006. Reactivity of soil acidity correctives and conditioners in lysimeters. Revista Brasileira de Ciência do Solo 30: 849-857 (in Portuguese, with abstract in English).

Sposito, G. 1989. The Chemistry of Soils. p. 277. Oxford University Press, New York, NY, USA.

Vance, G.F.; Stevenson, F.J.; Sikora, F.J. 1996. Environmental chemistry of aluminum organic complexes. p. 169-220. In: Sposito, G., ed. The environmental chemistry of aluminum. 2ed. Lewis, Boca Raton, FL, USA.

Wolt, J.D. 1994. Soil Solution Chemistry: Applications to Environmental Science and Agriculture. John Willey, New York, NY, USA. p.345.

Zambrosi, F.C.B.; Alleoni, L.R.F.; Caires, E.F. 2007. Nutrient concentration in soil water extracts and soybean nutrition in response to lime and gypsum applications to an acid Oxisol under no-till system. Nutrient Cycling in Agroecosystems 79: 169-179.

Zambrosi, F.C.B.; Alleoni, L.R.F.; Caires, E.F. 2008. Liming and ionic speciation of an Oxisol under no-till system. Scientia Agricola 65: 190-203. 